\title{
Nonprobabilistic uncertain model updating and optimization design of thermal protection system
}

\author{
Wenting Jiang ${ }^{\mathrm{a}}$, Xiaojun Wang ${ }^{\mathrm{b}, *}$, Ruixing Wang ${ }^{\mathrm{c}, \mathrm{d}, *}$, Qinghe Shi $^{\mathrm{e}}$, Jingjing Zhu ${ }^{\mathrm{b}}$ \\ ${ }^{a}$ Key Laboratory of Light-Duty Gas-Turbine, Institute of Engineering Thermophysics, Chinese Academy of Sciences, Beijing 100190, China \\ ${ }^{\mathrm{b}}$ Institute of Solid Mechanics, Beihang University, Beijing 100191, China \\ ${ }^{c}$ Key Laboratory for Mechanics in Fluid Solid Coupling Systems, Institute of Mechanics, Chinese Academy of Sciences, No. 15 Beisihuanxi Road, Beijing 100190, China \\ ${ }^{\mathrm{d}}$ School of Engineering Science, University of Chinese Academy of Science, Beijing 100049, China \\ ${ }^{\mathrm{e}}$ School of Materials and Engineering, Jiangsu University of Technology, Changzhou, Jiangsu 213001, China
}

\section{H I G H L I G H T S}

- An improved Latin hypercube design is proposed for correlated factors.

- The upper bound by uncertainty analysis is $5.26 \%$ higher than that by sampling.

- An uncertainty-based model updating method is proposed closer to test data.

- The nonprobabilistic TPS design is $3.44 \%$ lighter and verified by experiment.

\section{A R T I C L E I N F O}

\section{Keywords:}

Thermal protection system

Nonprobabilistic optimization

Sensitivity analysis

Uncertainty analysis

Model updating

\begin{abstract}
A B S T R A C T
Reusable launch vehicles are subjected to intense aerodynamic heating during the hypersonic re-entry stage. Thus, thermal protection system (TPS) design methods that consider uncertainty have become increasingly important in recent years. In this study, a nonprobabilistic TPS optimization design that takes into account deviations in temperature-dependent thermophysical property parameters is carried out with corresponding experimental verification. An improved Latin hypercube design (ILHD) is first proposed to solve the sampling problem in the case where the distribution domains of correlated uncertainty parameters interfere with each other. Based on the ILHD, uncertainty and sensitivity analyses of the TPS heat transfer are performed, in which the importance and effect trends of uncertainty parameters to responses are clearly identified. In terms of both computational costs and accuracy, the ILHD method has a significant advantage because of the excellent abilities of random sampling to satisfy certain constraints, space-filling, and nonlinear response-fitting. The ILHD's superiority in uncertainty and sensitivity analyses is also proved compared with response bounds by sampling. During experimental verification, an uncertainty-based model updating method is proposed to modify the heat transfer numerical model of test pieces. Finally, a lighter design is obtained and the correctness and validity of applied methods are verified.
\end{abstract}

\section{Introduction}

The thermal protection system (TPS) applied to the external surface of reusable launch vehicles must maintain the temperatures of the underlying material and internal structure within acceptable limits as well as satisfy the requirement of mechanical loads [1,2]. Inadequate thermal insulation performance may cause the failure of the TPS or even the vehicle itself because the temperatures exceed the specified limits [3]. Therefore, efficient TPS design is required to minimize the vehicle's weight and ensure its own thermal and mechanical integrity during re-entry using lightweight materials that provide high thermal protection and insulation [4].

The TPS is divided into three categories: passive, semi-passive, and active [5]. The passive TPS is considered the safest and most weightefficient [6], and has attracted the attention of researchers nationally and internationally. The passive TPS can be classified as a heat sink structure, hot structure, and insulated structure according to different thermal protection principles [5]. The insulated structure has the

\footnotetext{
* Corresponding authors at: Institute of Solid Mechanics, Beihang University, Beijing 100191, China (X. Wang); Key Laboratory for Mechanics in Fluid Solid Coupling Systems, Institute of Mechanics, Chinese Academy of Sciences, No. 15 Beisihuanxi Road, Beijing 100190, China (R. Wang).

E-mail addresses: xjwang@buaa.edu.cn (X. Wang), wangruixing@imech.ac.cn (R. Wang).
} 


\begin{tabular}{|c|c|c|c|}
\hline \multicolumn{3}{|c|}{ Nomenclature } & \multirow{3}{*}{$\begin{array}{l}\text { for each factor } \\
\text { the } j^{\text {th }} \text { level of } x_{k} \\
\text { the } j^{\text {th }} \text { level of } x_{k+1}\end{array}$} \\
\hline & & $x_{k}(j)$ & \\
\hline$T$ & temperature $(\mathrm{K})$ & $x_{k+1}(j)$ & \\
\hline$t$ & time $(s)$ & $x_{k}^{i}$ & upper bound of $i^{\text {th }}$ subinterval \\
\hline$z$ & spatial coordinate through the TPS thickness direction & $x_{k}^{i-1}$ & lower bound of $i^{\text {th }}$ subinterval \\
\hline$k_{i}$ & effective thermal conductivity of $i^{\text {th }}$-layer TPS $(\mathrm{W} / \mathrm{m} \cdot \mathrm{K})$ & $I$ & interference interval of $\left[x_{k+1}^{\min }, x_{k}^{\max }\right]$ \\
\hline$k_{i j}$ & the $j^{\text {th }}$ interpolation point of $k_{i}(\mathrm{~W} / \mathrm{m} \cdot \mathrm{K})$ & $r$ & number of $x_{k}(j)$ in $I$ \\
\hline$\rho_{i}$ & effective density of $i^{\text {th }}$-layer TPS $\left(\mathrm{kg} / \mathrm{m}^{3}\right)$ & $s$ & number of $x_{k+1}(j)$ in $I$ \\
\hline$\rho_{i j}$ & the $j^{\text {th }}$ interpolation point of $\rho_{i}\left(\mathrm{~kg} / \mathrm{m}^{3}\right)$ & $c$ & number of cycles \\
\hline$c_{i}$ & effective specific heat of $i^{\text {th }}$-layer TPS $(\mathrm{J} / \mathrm{kg} \cdot \mathrm{K})$ & $\phi$ & empty set \\
\hline$c_{i j}$ & the $j^{\text {th }}$ interpolation point of $c_{i}(\mathrm{~J} / \mathrm{kg} \cdot \mathrm{K})$ & $A_{1}$ & distribution interval of $T_{3-\max }$ by uncertainty analysis $(\mathrm{K})$ \\
\hline$T_{S}$ & TPS initial temperature $(\mathrm{K})$ & $A_{2}$ & distribution interval of $T_{5-\max }$ by uncertainty analysis $(\mathrm{K})$ \\
\hline$q$ & re-entry heat flux $\left(\mathrm{W} / \mathrm{cm}^{2}\right)$ & $B_{1}$ & distribution interval of $T_{3-\max }$ by sensitivity and un- \\
\hline$h_{\text {top }}$ & convection coefficient at TPS top surface $\left(\mathrm{W} / \mathrm{m}^{2} \cdot \mathrm{K}\right)$ & & certainty analyses (K) \\
\hline $\begin{array}{l}h_{\text {bottom }} \\
\varepsilon\end{array}$ & $\begin{array}{l}\text { convection coefficient at TPS bottom surface }\left(\mathrm{W} / \mathrm{m}^{2} \cdot \mathrm{K}\right) \\
\text { Emissivity }\end{array}$ & $B_{2}$ & $\begin{array}{l}\text { distribution interval of } T_{5-\max } \text { by sensitivity and un- } \\
\text { certainty analyses }(\mathrm{K})\end{array}$ \\
\hline$\sigma$ & Stefan-Boltzmann constant $\left(\mathrm{W} / \mathrm{m}^{2} \cdot \mathrm{K}^{4}\right)$ & $C_{1}$ & distribution interval of $T_{3-\max }$ by sampling (K) \\
\hline$l$ & TPS' total thickness (mm) & $C_{2}$ & distribution interval of $T_{5-\max }$ by sampling $(\mathrm{K})$ \\
\hline$l_{3}$ & thicknesses of outer insulation (mm) & $T_{3-\max }^{\text {upper }}$ & upper bound of $T_{3-\max }(\mathrm{K})$ \\
\hline$l_{4}$ & thicknesses of inner insulation (mm) & $T_{5-\max }^{\text {upper }}$ & upper bound of $T_{5-\max }(\mathrm{K})$ \\
\hline mass & TPS' mass $(\mathrm{g})$ & $T_{3}$-failure & failure temperature of inner insulation $(\mathrm{K})$ \\
\hline$T_{3-\max }$ & maximum temperature of inner insulation $(\mathrm{K})$ & $T_{5}$ - failure & failure temperature of skin $(\mathrm{K})$ \\
\hline$T_{5-\max }$ & maximum temperature of skin $(\mathrm{K})$ & $T_{\mathrm{e}}$ & experimental heating load $(\mathrm{K})$ \\
\hline$T_{3-\text { allow }}$ & allowable temperature of inner insulation (K) & $t_{\mathrm{e}}$ & experimental heating time (s) \\
\hline$T_{5-\text { allow }}$ & allowable temperature of skin $(\mathrm{K})$ & $t_{\mathrm{c}}$ & numerical calculation time (s) \\
\hline$n$ & number of DOE factors & $T_{6}-\max$ & maximum temperature of TPS bottom surface $(\mathrm{K})$ \\
\hline$x_{k}$ & the $k^{\text {th }}$ factor & $t_{T_{6-\max }}$ & corresponding time of $T_{6-\max }(\mathrm{s})$ \\
\hline$x_{k+1}$ & the $k+1^{\text {th }}$ factor & $T_{6-\max }^{\mathrm{FEM}-\mathrm{MED}}$ & interval median of $T_{6-\max }$ by finite element analysis (K) \\
\hline$x_{k}^{\min }$ & minimum value of $x_{k}$ & $t_{T_{6-\max }}^{\mathrm{FEM}-\mathrm{MED}}$ & interval median of $t_{T_{6-\max }}$ by finite element analysis (s) \\
\hline$x_{k+1}^{\min }$ & minimum value of $x_{k+1}$ & $T_{6-\max }^{\mathrm{EXP}}$ & $T_{6-\max }$ for test data $(\mathrm{K})$ \\
\hline$x_{k}^{\max }$ & maximum value of $x_{k}$ & $t_{T_{6-\max }^{\mathrm{EXP}}}^{\mathrm{EX}}$ & $t_{T_{6-\max }}$ for test data $(\mathrm{s})$ \\
\hline$x_{k}^{\mathrm{mid}}$ & interval median of $x_{k}$ & $\omega_{1}$ & the first weighting factor \\
\hline$x_{k+1}^{\operatorname{mid}}$ & interval median of $x_{k+1}$ & $\omega_{2}$ & the second weighting factor \\
\hline$m$ & number of levels, also number of distribution & & \\
\hline
\end{tabular}

advantages of both the heat sink structure and hot structure. It removes most of the heat via thermal radiation and absorbs only a small amount of the remaining heat, which is conducted through the insulation to the inner structure and stored in the form of heat sinks. Typical insulated structures include flexible ceramic blankets, rigid ceramic tiles, metallic panels, etc. [7], all of which have multilayer features that make TPS design difficult, especially the processes of advanced uncertainty analysis and optimization. The reason is the thermal insulation efficiency determined by both the effective thermal conductivity and thermal storage of the multilayer structures [8].

The uncertainty in TPS design has many sources and cannot be ignored. The primary sources are: stochastic variability, including natural fluctuations in atmospheric conditions and surface texture variations of TPS materials; structural uncertainty caused by simplified physical model or its numerical discretization; and parametric uncertainty arising from the input model parameters [9]. Despite a number of theoretical achievements in the deterministic analysis and optimization design of TPS [10-14], there are few developments in TPS uncertain design. Historical approaches for dealing with uncertainties depend on expert judgment to assign uncertainty levels to factors affecting TPS sizing [15]. Thereafter, the uncertainty design of TPS is usually performed in the two following methods: Statistical Error Analysis (SEA) and Monte Carlo Simulation (MCS) [16,17]. Dec and Mitcheltree first applied the MCS to determine the TPS margins of the Mars Sample Return Earth Entry Vehicle by a probabilistic design approach with inevitably high computational costs [18]. The SEA has the lower computational costs than the MCS, however, its calculation accuracy is not as good as the latter. Aiming at the above problem, Gomez-San-
Juan et al. proposed an intermediate method to balance the computational costs and calculation accuracy between SEA and MCS [16]. In the above-mentioned researches on the uncertainty design of TPS, on the one hand, the temperature dependence and correlation of uncertain input parameters are often ignored, which has failed to result in a highreliability TPS; on the other hand, the high computational costs associated with uncertainty analysis using the MCS or SEA must also be taken into account. Hence, a refined TPS uncertainty design that can effectively assess and consider the impact of input-dependent uncertainties on output responses must be developed with a lower computational cost.

For a complex structure, the errors between calculated values obtained by numerical analysis and true value are enormous [19]. Thus, eliminating or reducing these deviations is a precondition of TPS reliability design Model updating technique can help realize this precondition [20]. Model updating methods can be divided into two major groups: the direct method and the iterative method [21]. The latter can be formulated as a mathematical optimization model by sensitivity analysis and computational intelligence techniques, and is more likely to be adopted. In terms of the thermal mathematical model updating, Torralbo and Sanz-Andres et al. have done a lot of work [22-23]. However, there are very few researches on model updating in TPS transient heat transfer analysis considering the uncertainty of input parameters, which restricts the high-performance design of TPS to a large extent.

In view of the above-mentioned problems, and taking a typical TPS as the research object, the focus of this study is illustrated in Fig. 1. Firstly, an improved Latin hypercube design is developed and applied to 


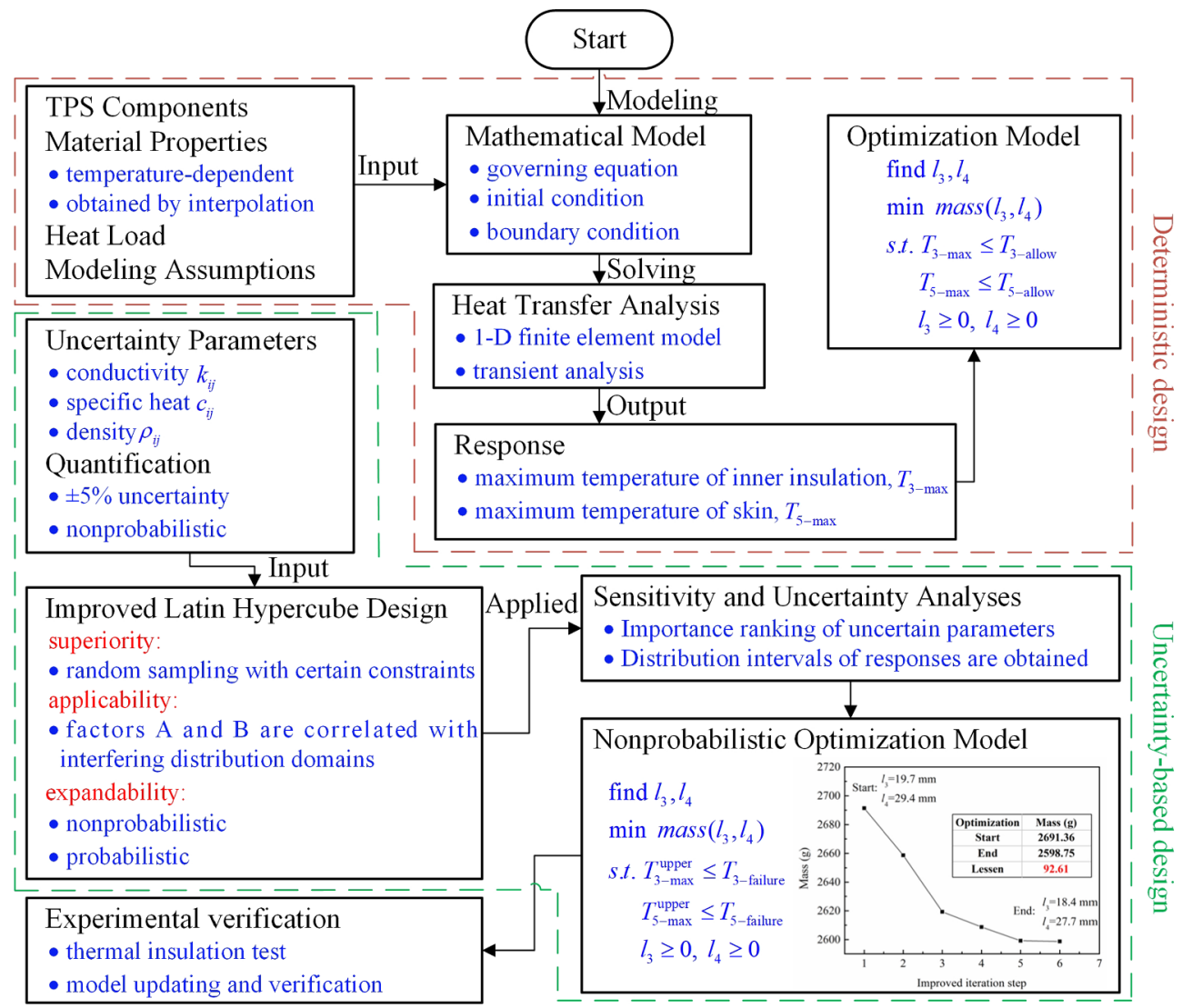

Fig. 1. Flow chart of the study.

the uncertainty and sensitivity analyses of TPS. The novelty of the approach is that it takes into account the correlations between the temperature-dependent characteristics of the same kind of uncertainty input variables. The relationships between uncertainty input variables and output responses are identified, and the contributions of each uncertainty input variable are ranked. Secondly, a complete nonprobabilistic optimization process, which is feasible for TPS, is established by defining the thickness of each component as a design variable as well as considering the temperature constraints, which significantly reduce the computation time. Thirdly, an experiment on TPS thermal insulation performance is used to verify all the proposed approaches, and an uncertainty-based model updating approach for TPS heat transfer analysis is proposed. The novelty of the approach is that it directly selects the key responses related to the failure of a TPS or even the entire vehicles as the objectives to be updated. Simultaneously, the variables also depend on the service environment of the TPS. Finally, it is shown that excellent results were achieved.

\section{Deterministic design}

\subsection{Description and assumptions of the model}

Fig. 2 shows a typical insulated TPS with six layers of components consisting of, from to bottom, a carbon/carbon (C/C) panel, flexible fabric, outer insulation, inner insulation, strain isolation pad, and loadbearing skin, all bonded together with room-temperature vulcanizing adhesive. As the external surface of the TPS, the outer surface of the C/ $\mathrm{C}$ panel is directly subjected to the severe aerodynamic heating during re-entry. At this stage, only a small portion of the remaining heat is transferred to the TPS interior, with a significant amount of heat radiated to the environment.

The initial thickness and effective thermophysical property parameters of the TPS are listed in Table 1. Among them, the temperature- dependent specific heat and thermal conductivities were obtained by linear interpolation [4]. The interpolation points involved and curves are shown in Fig. 3.

Combined heat transfer modes exist in the re-entry progress of a reusable launch vehicle for an insulated TPS, including radiation, solid heat conduction through each component, gas conduction and convection [1]. For the convenience of TPS optimization design, the following assumptions were made $[24,25]$ :

(1) Heat is transferred only in the thickness direction of the TPS. The in-plane temperature gradients are small enough to be negligible.

(2) The effect of the adhesive on heat transfer is ignored.

(3) The contact resistances among substructures are ignored.

(4) No ablation occurs at the top surface of the TPS.

(5) No heat transfer occurs at the bottom surface of the TPS.

(6) The initial temperature of the TPS is the same as the ambient temperature.

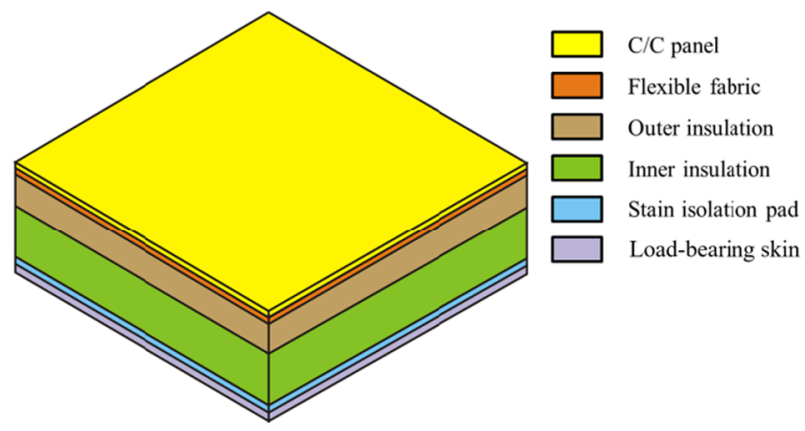

Fig. 2. Components of a typical TPS. 
Table 1

Initial dimensions and thermophysical properties for TPS.

\begin{tabular}{|c|c|c|c|c|c|c|}
\hline Component layer & first & second & third & fourth & fifth & sixth \\
\hline Thickness (mm) & 4 & 3 & 20 & 30 & 3 & 3 \\
\hline Density $\left(\mathrm{kg} / \mathrm{m}^{3}\right)$ & 1800 & 170 & 360 & 330 & 150 & 1650 \\
\hline Specific heat $(\mathrm{J} / \mathrm{kg} \cdot \mathrm{K})$ & Fig. 3 (a) & 1000 & Fig. 3 (a) & Fig. 3 (a) & 1000 & Fig. 3 (a) \\
\hline Thermal conductivity $(\mathrm{W} / \mathrm{m} \cdot \mathrm{K})$ & Fig. 3 (b) & Fig. 3 (b) & Fig. 3 (b) & Fig. 3 (b) & 0.03 & Fig. 3 (b) \\
\hline
\end{tabular}

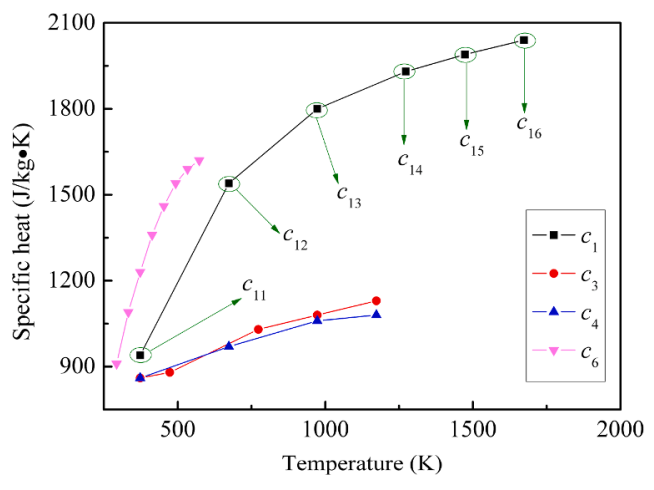

(a) Specific heat

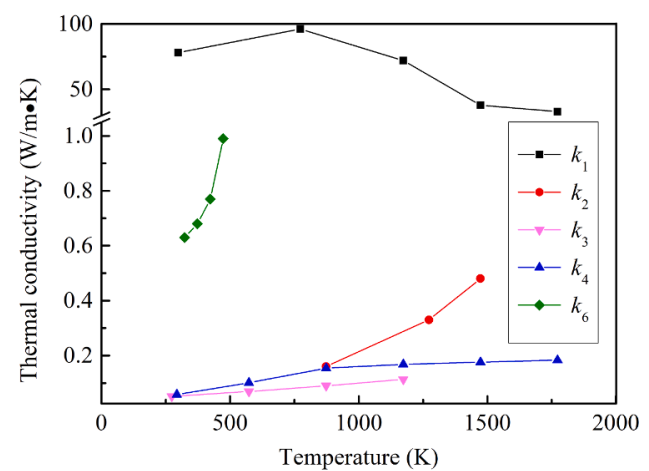

(b) Thermal conductivity

Fig. 3. Temperature-dependent thermophysical property parameters.

\subsection{Transient heat transfer equations and analysis}

Based on the above assumptions, the temperature field analysis of the TPS was converted into solving a one-dimensional transient heat transfer problem. Taking the external surface of the TPS as the origin, a one-dimensional Cartesian coordinate system was established along the thickness direction. Inside each component (i.e. each layer of the TPS), the governing equation for conservation of energy can be expressed as:

$\frac{\partial}{\partial z}\left(k_{i}(T) \frac{\partial T(z, t)}{\partial z}\right)-\rho_{i}(T) c_{i}(T) \frac{\partial T(z, t)}{\partial t}=0$

where index $i$ denotes the $i^{\text {th }}$ layer of the TPS, $(i=1,2 \cdots, 6), z$ is the spatial coordinate along the TPS thickness direction, and $t$ is the time. $T$ is the TPS temperature, which is a binary function of $z$ and $t . k_{i}, \rho_{i}$, and $c_{i}$ are the effective thermal conductivity, density, and specific heat of the $i^{\text {th }}$ component, respectively, some of which are functions of temperature. At the interface between the two components, the temperature and heat flux were set to be continuous.

Owing to the assumption that the bottom surface of the TPS is adiabatic, the boundary condition only illustrates the heat transfer at the top surface of the TPS, which can be divided into two stages.

Stage 1: Heat is applied to the top surface of TPS by heat flux and dissipated by radiation during re-entry.

Stage 2: Heat is dissipated at the top surface of TPS by radiation and convection after landing.

Thus, Eq. (1) is subjected to the initial and boundary conditions as follows:

$T(z, 0)=T_{s}$

$-\left.k_{i} \frac{\partial T(0, t)}{\partial z}\right|_{i=1}=\left\{\begin{array}{c}q(t)-\varepsilon(T) \sigma\left[T^{4}(0, t)-T_{s}^{4}\right] \rightarrow \text { Stage 1 } \\ -h_{\text {top }}\left[T(0, t)-T_{s}\right]-\varepsilon(T) \sigma\left[T^{4}(0, t)-T_{s}^{4}\right] \rightarrow \text { Stage } 2\end{array}\right.$

where $T_{s}$ is the initial temperature of the TPS, which was assumed as $288 \mathrm{~K} ; q$ is a typical re-entry aerodynamic heating load applied to the top surface of the TPS; $h_{\text {top }}$ is the convection coefficient shown in Fig. 4 [26]; $\varepsilon$ is the emissivity which is a function of temperature, and it is simplified as a constant of 0.85 in the simulation analysis; and $\sigma$ is the Stefan-Boltzmann constant of $5.67 \times 10^{-8} \mathrm{~W} /\left(\mathrm{m}^{2} \cdot \mathrm{K}^{4}\right)$.

A simplified mathematical model of the TPS heat transfer has been completed. The model was discretized by a commercial finite element analysis software, as shown in Fig. 5. Further, the transient temperature field of the TPS can be acquired.

Some temperature responses of interest are shown in Fig. 6. Among them, the temperature of top surface represents the radiation equilibrium temperature during the re-entry stage, which depends on the reentry heat flux and the emissivity instead of structural thickness. Maximum radiation equilibrium temperature is $1450 \mathrm{~K}$ less than the allowable temperature of the outer insulation, that is, the outer insulation is not at risk of temperature failure. Thus, the responses that need to be considered and strictly controlled in the design stage are the inner insulation and the skin's maximum temperatures.

\subsection{Deterministic optimization}

The overall goal of TPS design is to minimize the vehicle's weight while preventing its structure from exceeding its design temperature limit during re-entry.

As a result of the vehicle' sensitivity to weight, the optimization objective is to minimize the TPS mass. The thicknesses of the outer and inner insulation were taken as design variables because a certain

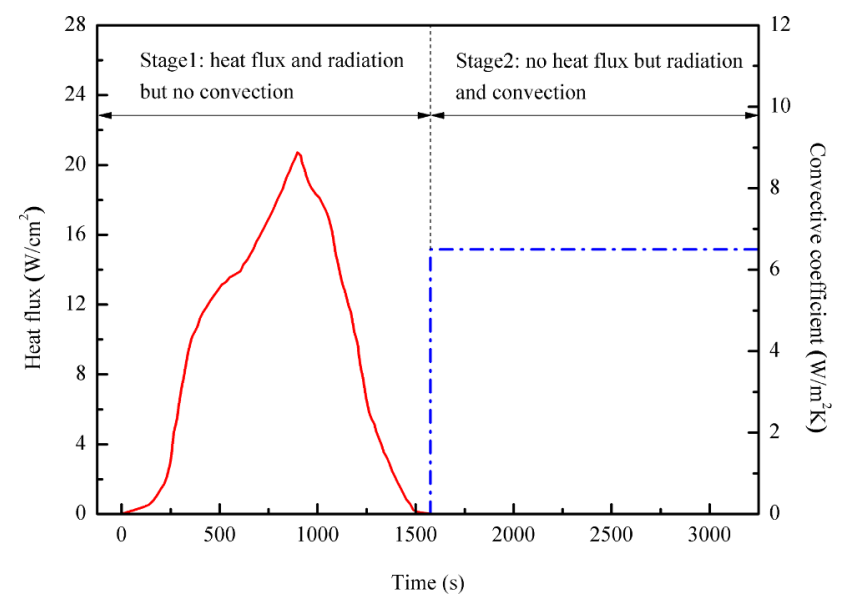

Fig. 4. A typical re-entry aerodynamic heating condition. 


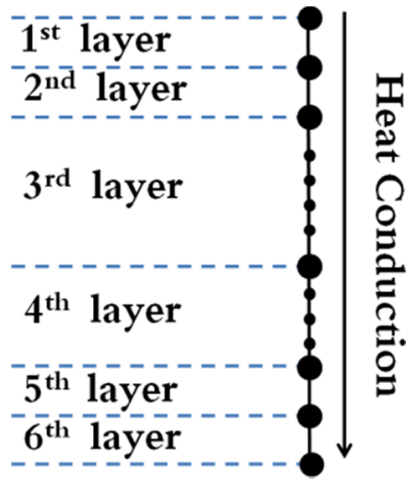

Fig. 5. One-dimensional finite element model.

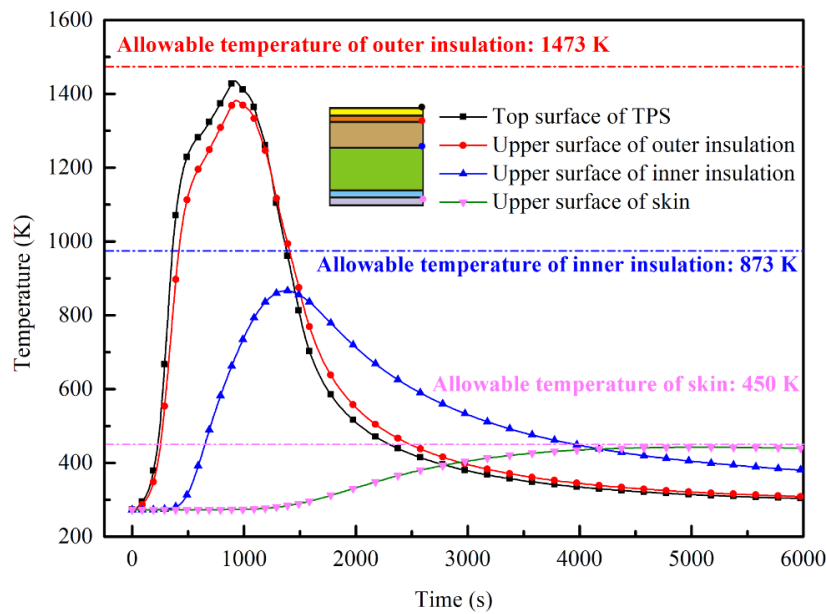

Fig. 6. Time-dependent temperature responses of interest.

Table 2

Failure thresholds, allowable values and their safety margins.

\begin{tabular}{llll}
\hline Component & Allowable value & Safety margin & Failure threshold \\
\hline Inner insulation & $873 \mathrm{~K}$ & $76.64 \mathrm{~K}$ & $949.74 \mathrm{~K}$ \\
Skin & $450 \mathrm{~K}$ & $37.15 \mathrm{~K}$ & $487.15 \mathrm{~K}$ \\
\hline
\end{tabular}

thickness of insulation is needed to ensure adequate thermal insulation performance. Except for the inner insulation and the skin, the allowable temperatures for all remaining components are higher than their maximum temperatures in practice. Therefore, the constraint condition is that the maximum temperatures of the inner insulation and the skin

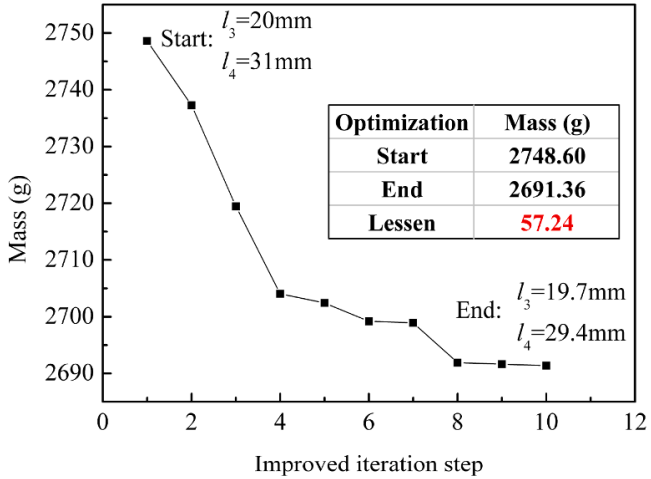

(a) Mass

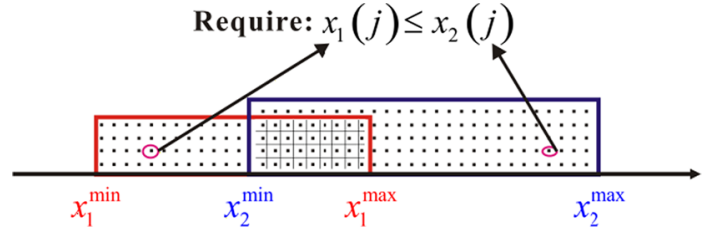

Fig. 8. Correlated factors of $x_{1}$ and $x_{2}$.

must not exceed their allowable temperatures respectively. Moreover, the maximum temperature of the inner insulation must occur at the interface between the outer insulation and inner insulation, while the maximum temperature of the skin must occur at the interface between the strain isolation pad and skin.

Therefore, the deterministic optimization model of the TPS can be mathematically expressed as

$$
\begin{gathered}
\text { find } l_{3}, l_{4} \\
\min \operatorname{mass}\left(l_{3}, l_{4}\right) \\
\text { s. } t . T_{3-\max } \leqslant T_{3 \text {-allow }} \\
T_{5-\max } \leqslant T_{5 \text {-allow }} \\
l_{3} \geqslant 0, l_{4} \geqslant 0
\end{gathered}
$$

where, $l_{3}$ and $l_{4}$ are the design variables, that denote the thickness of outer and inner insulation, respectively; mass represents the TPS mass function of $l_{3}$ and $l_{4} ; T_{3-\max }$ and $T_{5-\max }$ are the maximum temperatures of the inner insulation and skin; and $T_{3 \text {-allow }}$ and $T_{5 \text {-allow }}$ are the allowable temperatures of the inner insulation and skin. The failure thresholds, i.e., the allowable values and safety margin for each constraint, are listed in Table 2. The safety margins can be considered as the risks allocated for each failure mode [27].

After the solution using the genetic algorithm, the design variable values obtained by deterministic optimization are $l_{3}=19.7 \mathrm{~mm}$ and $l_{4}=29.4 \mathrm{~mm}$; and the corresponding structural mass is $2691.36 \mathrm{~g}$. The iteration histories of the TPS mass and two constrained responses are shown in Fig. 7. Here, the deterministic optimization is the basis of uncertainty-based design, and the former can provide a contrast for the latter.

\section{Uncertainty-based design}

\subsection{Improved Latin hypercube design}

The design of experiments (DOE) has advantages in constructing the approximate model, identifying key factors, determining the best combination of factors and analyzing the relationship and trends between factors and responses. In DOE, "factor" and "response" are the technical terms used for the input and output parameter, respectively; and "level" is the technical term used for the specific value of the factor.

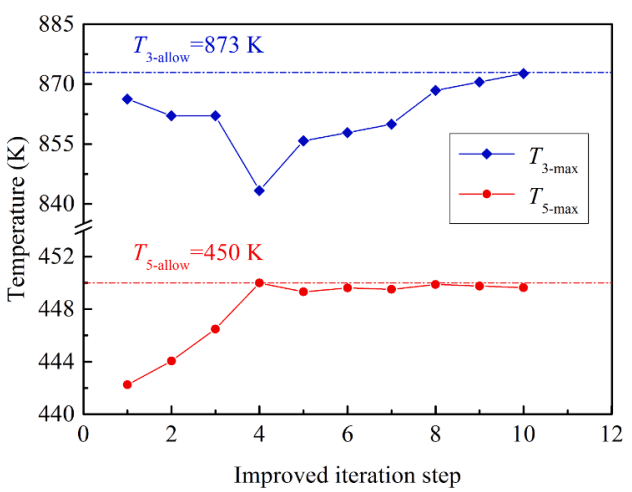

(b) Constrained responses

Fig. 7. Iteration history. 


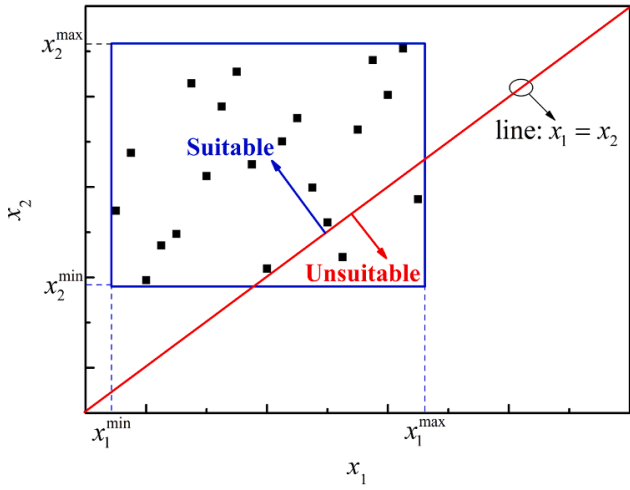

(a) LHD

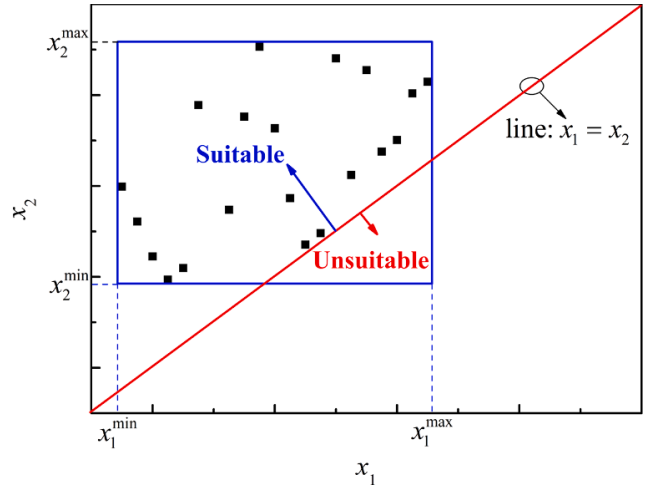

(b) ILHD

Fig. 9. Samples of $x_{1}$ and $x_{2}$.

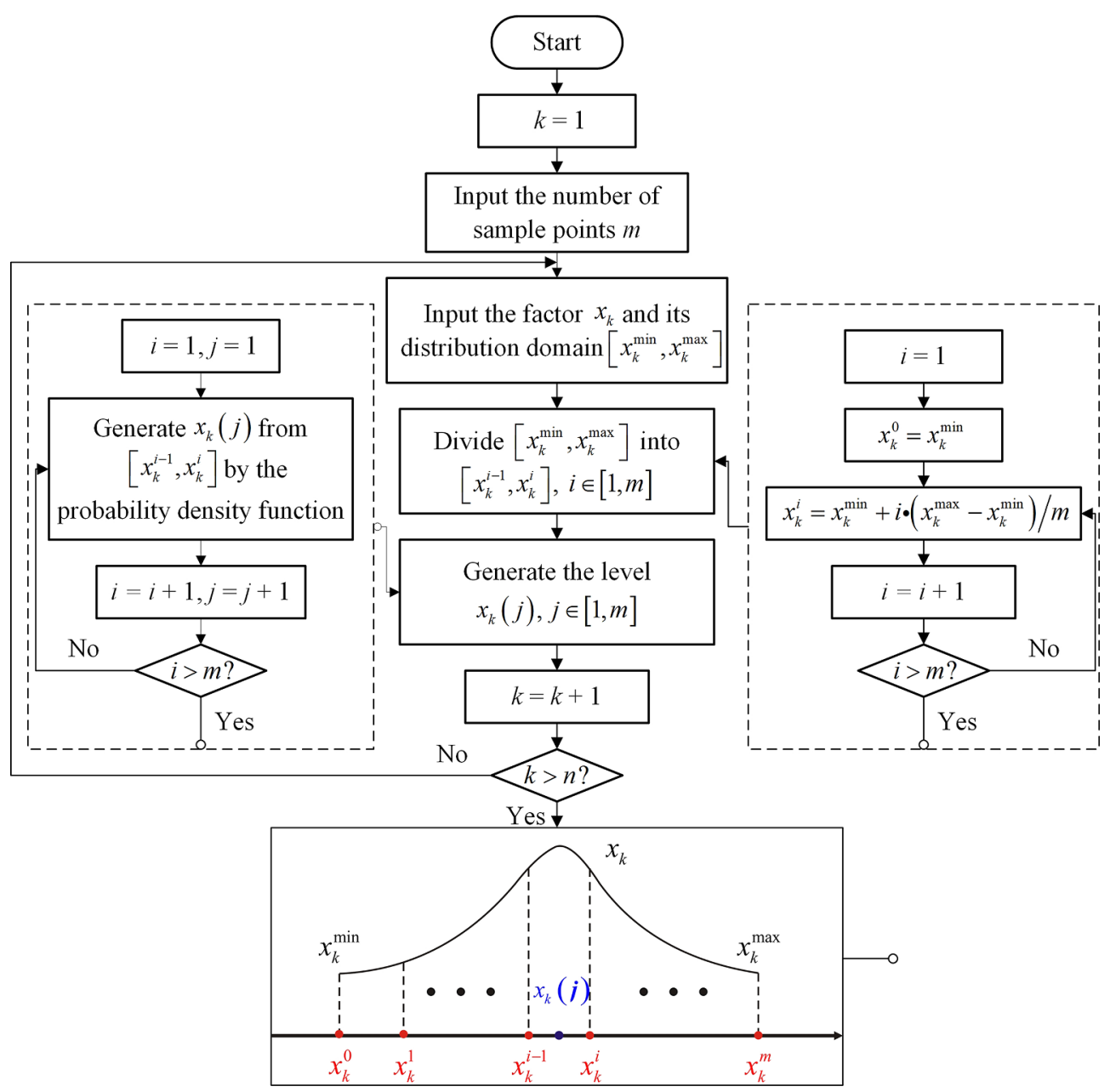

Fig. 10. Flow chart of first three steps for ILHD.

The distribution domain of each factor is an interval where the specific value defined as a level is extracted. The combination of each factor's level is known as a sample.

The Latin hypercube design (LHD) is one of the DOE methods with the excellent space-filling and nonlinear response-fitting abilities. However, LHD has limited applicability because it is only suitable in situations where the factors are independent of each other. In cases where the factors are not mutually independent, i.e. are related to each other, a specific correlation rule must be introduced to the LHD. Consequently, the improved LHD (ILHD) is proposed.

ILHD's superiority lies in the fact that it takes into account of the correlation between factors in addition to retaining the LHD's whole advantages. For example, as shown in Fig. 8, factors $x_{1}$ and $x_{2}$ are correlated, and their levels (i.e., $x_{1}(j)$ and $\left.x_{2}(j)\right)$ must comply with the rule $x_{1}(j) \leqslant x_{2}(j)$. Meanwhile, the distribution domains of factors $x_{1}$ and $x_{2}$ are interrelated. If LHD is used, then the unsuitable sample of $\left(x_{1}(j), x_{2}(j)\right)$, where $x_{1}(j)>x_{2}(j)$, will be generated incorrectly owing to the LHD's completely random sampling, as illustrated in Fig. 9 (a). On the other hand, if ILHD is employed, all samples that meet the requirement $x_{1}(j) \leqslant x_{2}(j)$, owing to ILHD's conditionally random sampling, are suitable, as shown in Fig. 9 (b).

The detailed procedures of ILHD are outlined as follows: 


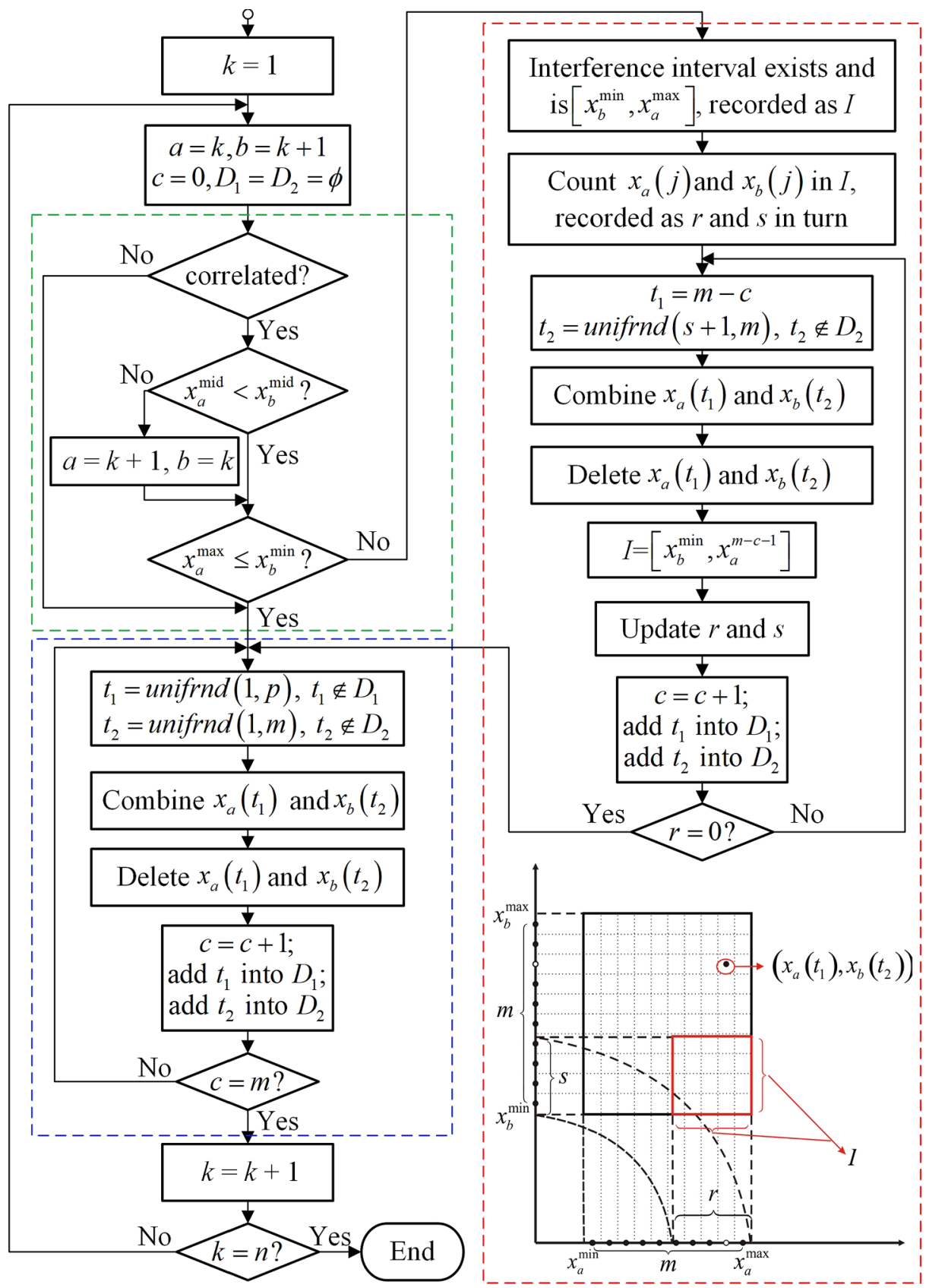

Fig. 11. Flow chart of the other steps for ILHD.

Step 1: Select the factor $x_{k}$ with its distribution interval of $\left[x_{k}^{\min }, x_{k}^{\max }\right], k \in[1, n]$.

Step 2: Divide $\left[x_{k}^{\min }, x_{k}^{\max }\right]$ into $m$ subintervals of $\left[x_{k}^{i-1}, x_{k}^{i}\right], i \in[1, m]$, evenly.

Step 3: Generate the level $x_{k}(j)$ from $\left[x_{k}^{i-1}, x_{k}^{i}\right]$ by the probability density function of $x_{k}$, where $j=i \in[1, m]$.

The above three steps are consistent with the LHD method, as shown in Fig. 10. The following steps for ILHD are illustrated in Fig. 11.

Step 4: Determine whether $x_{k}$ and $x_{k+1}$ are correlated or not. If yes, perform Step 6.

Step 5: Combine the residual levels of $x_{k}$ and $x_{k+1}$ randomly.

Step 6: Compare interval medians of $x_{k}^{\text {mid }}$ and $x_{k+1}^{\text {mid }}$. If $x_{k}^{\text {mid }} \geqslant x_{k+1}^{\text {mid }}$, then exchange $x_{k}$ and $x_{k+1}$.

Step 7: Compare $x_{k}^{\max }$ and $x_{k+1}^{\min }$. If $x_{k}^{\max }$ is not greater than $x_{k+1}^{\min }$, then perform Step 5.
Step 8: Record the interference interval $\left[x_{k+1}^{\min }, x_{k}^{\max }\right]$ as $I$, and count the levels of $x_{k}$ and $x_{k+1}$ in $I$, recorded as $r$ and $s$ in turn.

Step 9: Let $c=0$, where $c$ is the number of cycles.

Step 10: Select $x_{k+1}\left(t_{2}\right)$ randomly, $t_{2} \in[s+1, m]$, and then combine $x_{k+1}\left(t_{2}\right)$ and $x_{k}(r)$.

Step 11: Delete the subinterval $\left[x_{k}^{m-c}, x_{k}^{m-c+1}\right]$ in $I$, add this subinterval into the non-interference interval, and then update the $I$, recorded as $\left[x_{k+1}^{\min }, x_{k}^{m-c}\right]$.

Step 12: Delete $x_{k+1}\left(t_{2}\right)$, and then update $s$ and $r$.

Step 13: Let $c=c+1$.

Step 14: Perform Steps 9-13 repeatedly until $r=0$; then perform Step 5.

Step 15: Perform Steps 4-14 from $k=1$ to $k=n-1$ in turn, and then end.

Thus far, a set of samples under the correlation constraint has been obtained, recorded as $m \times n$ ILHD. Here, $n$ is the number of factors; $m$ is 


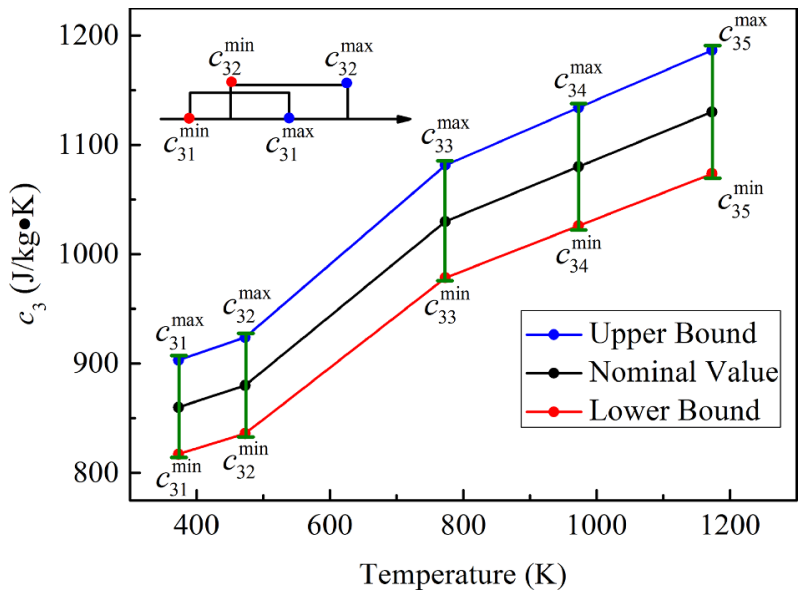

Fig. 12. Bounds of the specific heat for the outer insulation.

the number of levels; and the samples are combinations of different levels of $n$ factors, regarded as the points of $n$-dimensional space, whose number is also $m$. Next, ILHD is applied to the uncertainty-based design of TPS.

\subsection{Sensitivity and uncertainty analyses based on ILHD}

Nonprobabilistic interval analysis is suitable for dealing with imprecise uncertainties to predict the range of responses, which has much lower computational costs compared with the probability analysis [28-31]. In this section, the sensitivity and uncertainty analyses based on the ILHD of the TPS are studied with the input uncertainty parameters regarded as the nonprobabilistic interval data.

Before performing the sensitivity and uncertainty analyses, it is necessary to define and quantify the uncertainty parameters. The uncertainty parameters were set as the thermophysical property parameters of each component, including the density $\rho_{i j}$, specific heat $c_{i j}$, and thermal conductivity $k_{i j}$ of the $i^{\text {th }}$ component at their interpolation points; their estimated uncertainties were identified as $\pm 5 \%$. For example, the upper and lower bounds of the specific heat for the outer insulation at its interpolation points are shown in Fig. 12. It can be seen that the correlation constraints of $c_{31}(j) \leqslant c_{32}(j)$ and $c_{33}(j) \leqslant c_{34}(j) \leqslant c_{35}(j)$ exist and must be satisfied. Using traditional LHD, some sample points depicted in Fig. 13 (a) violate the law of $c_{31}(j) \leqslant c_{32}(j)$. In contrast, all the sample points generated by ILHD in Fig. 13 (b) satisfy the above correlation constraints, demonstrating illustrates the superiority of ILHD.

The approximate modeling is the basis of sensitivity and uncertainty analyses. This was also achieved using ILHD. First, all the uncertainty parameters with the number of 54 were regarded as the factors of ILHD. Next, a sample matrix consisting of all the sample points was generated by running the ILHD sampling code. A sample point was taken from the sample matrix as the input parameters one by one, then the transient temperature field analysis of the TPS was carried out, and $T_{3-\max }$ and $T_{5 \text {-max }}$ were exported to their response matrices. Lastly, using the response surface method, two accurate approximation models were successively constructed and confirmed, which describe the quantitative relationship between uncertainty parameters and responses of $T_{3-\max }$ and $T_{5-\max }$.

On this basis, the global sensitivity analysis (also known as importance measure analysis [32,33]) is presented by Parote graph which represents the contribution rates of the normalized uncertainty parameter to the response [34]. The contribution rates of uncertainty parameters to $T_{3-\max }$ and $T_{5-\text { max }}$ in the top 20 are shown in sequence in Fig. 14 and Fig. 15 by arranging the contribution rates in descending order. The uncertainty parameters with contribution rates less than $1 \%$ were ignored; hence, 17 and 19 key uncertainty parameters were selected for $T_{3-\max }$, and $T_{5-\max }$, respectively.

The effects of uncertainty parameters trends on $T_{3-\max }$ and $T_{5-\max }$ are identified in Fig. 16 and Fig. 17.

According to the effects of uncertainty parameters trends on responses, the combinations of levels were determined by inputting the bounds of $T_{3-\max }$ and $T_{5-\max }$ that can be obtained. By uncertainty analysis based on ILHD, the response intervals of $A_{1}$ and $A_{2}$ consisted of the bounds of $T_{3-\max }$ and $T_{5-\max }$, respectively, considering all the uncertainty parameters; meanwhile, the response intervals of $B_{1}$ and $B_{2}$ consisted of the bounds of $T_{3-\max }$ and $T_{5-\max }$, respectively, considering merely key uncertainty parameters; besides, the response distribution domains by sampling were recorded as $C_{1}$ and $C_{2}$, as listed in Table 3. On the one hand, $A_{1} \supset C_{1}$ and $A_{2} \supset C_{2}$, which prove the validity of the uncertainty analysis. $A_{1}$ and $A_{2}$ are respectively much wider than $C_{1}$ and $C_{2}$, and the upper bounds of $A_{1}$ and $A_{2}$ are respectively $2.82 \%$ and $5.26 \%$ higher than that of $C_{1}$ and $C_{2}$, which demonstrates the effectiveness of the uncertainty analysis. On the other hand, $A_{1} \supset B_{1}$ and $A_{2} \supset B_{2}$, which prove the validity of the sensitivity analysis. In addition, the width of $B_{1}$ and $B_{2}$ is respectively very close to that of $A_{1}$ and $A_{2}$, which demonstrates the effectiveness of the sensitivity analysis.

\subsection{Nonprobabilistic optimization}

In traditional deterministic optimization, with the safety margins considered as the risk allocated for each failure mode, designs that are either too conservative or too dangerous are realized owing to the rough estimate of uncertainty. However, uncertainty-based optimization meticulously takes into account the effects of uncertainty parameters on responses, which leads to a safer and lighter design.

The nonprobabilistic optimization model of the TPS can be

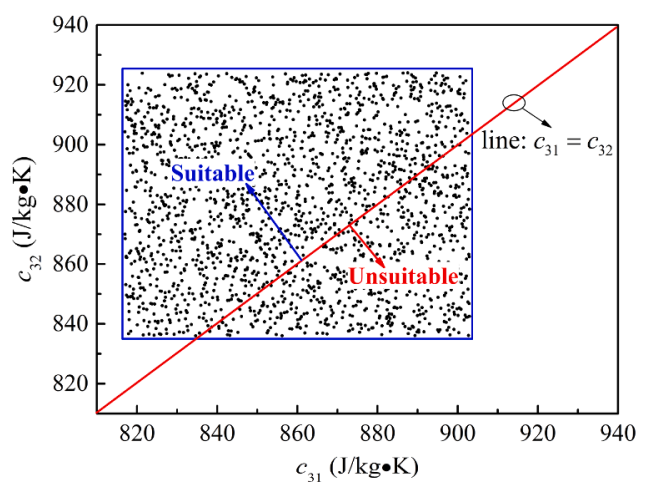

(a) LHD

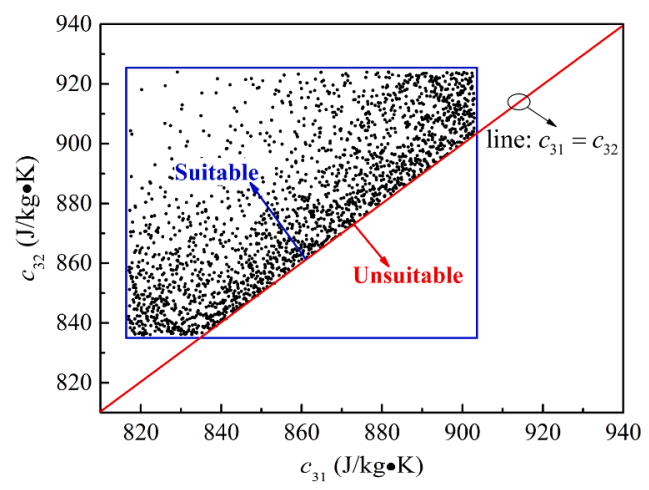

(b) ILHD

Fig. 13. Sample combinations of $c_{31}$ and $c_{32}$ generated. 


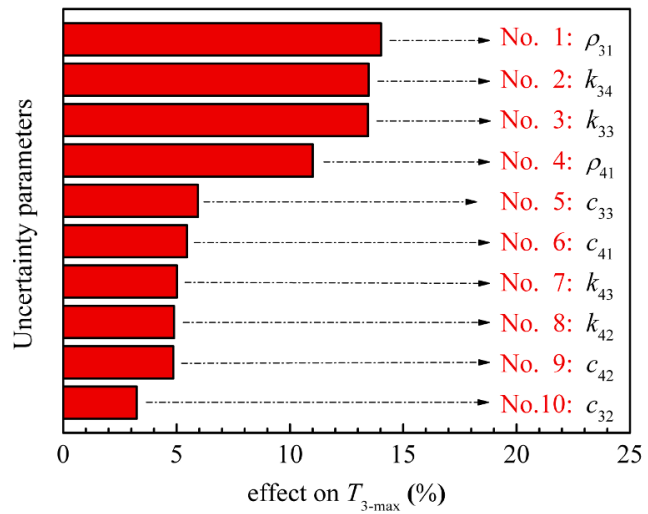

(a) Top 10

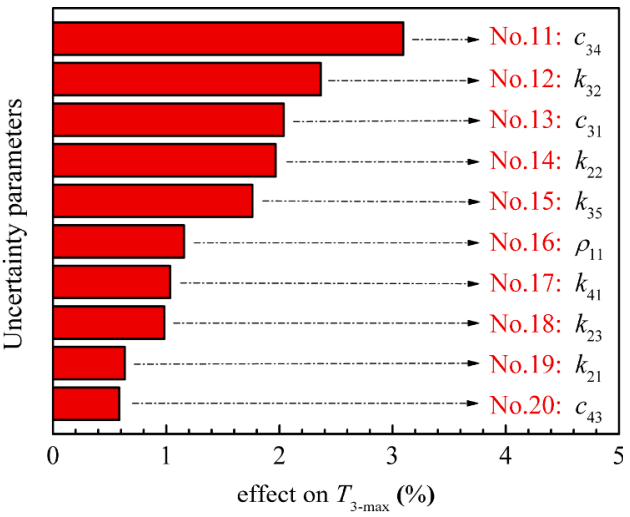

(b) Top 20

Fig. 14. Contribution rates of uncertainty parameters to $T_{3-\max }$.

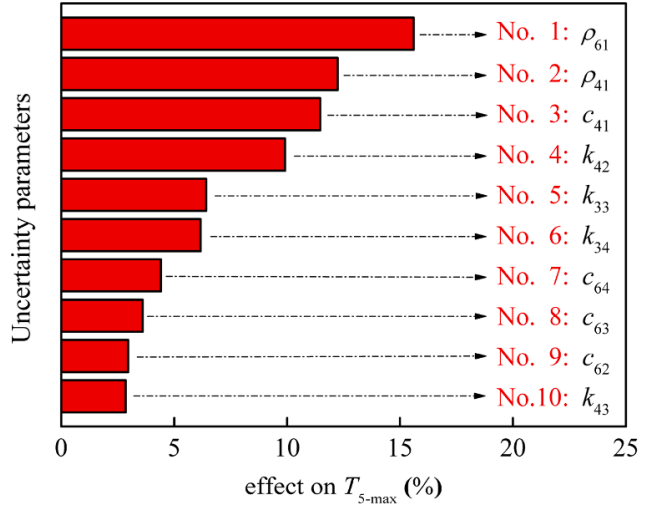

(a) Top 10

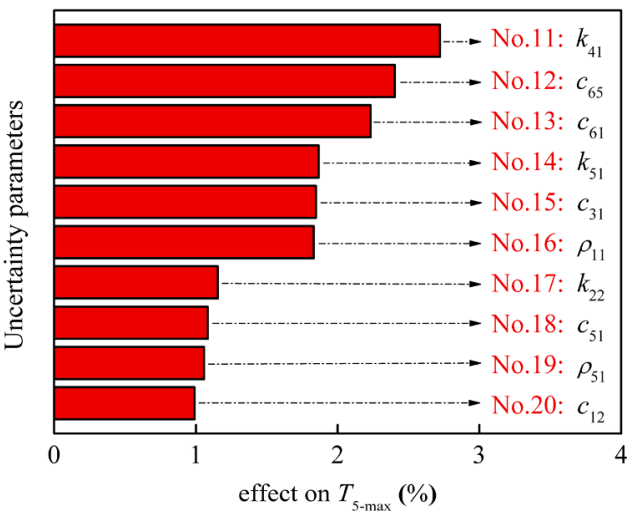

(b) Top 20

Fig. 15. Contribution rates of uncertainty parameters to $T_{5-\max }$.

mathematically expressed as

$$
\begin{gathered}
\text { find } l_{3}, l_{4} \\
\text { min } \operatorname{mass}\left(l_{3}, l_{4}\right) \\
\text { s. } t . T_{3-\max }^{\text {upper }} \leqslant T_{3} \text { - failure } \\
T_{5-\text { max }}^{\text {upper }} \leqslant T_{5 \text { - failure }} \\
l_{3} \geqslant 0, l_{4} \geqslant 0
\end{gathered}
$$

where, mass is the function that represents the nominal value of the TPS mass; $T_{3-\max }^{\text {upper }}$ and $T_{5-\max }^{\text {upper }}$ are the upper bounds of the inner insulation and the skin's maximum temperatures respectively, obtained by the uncertainty analysis of key uncertainty parameters; and $T_{3}$-failure and $T_{5 \text {-failure }}$ are the corresponding failure temperatures of the inner insulation and skin, respectively.

After the solution using the genetic algorithm, the final values of the design variables by nonprobabilistic optimization are $l_{3}=18.4 \mathrm{~mm}$ and $l_{4}=16.8 \mathrm{~mm}$; the corresponding structural mass is $2598.75 \mathrm{~g}$. The uncertainty-based design is $92.61 \mathrm{~g}$ lighter than the deterministic design. The iteration histories of the TPS mass and two constrained responses are shown in Fig. 18.

\section{Experimental verification}

\subsection{Experiment description}

A thermal insulation performance experiment was conducted with the two types of test pieces including the initial TPS prior to deterministic optimization and the final TPS after nonprobabilistic uncertainty optimization, to verify the correctness of the uncertainty analysis and optimization design for TPS. One of the TPS test pieces is shown in Fig. 19.

Because the bottom surface of the TPS test piece was exposed to an enclosure during the testing process, the assumption that no heat transfer occurs at the bottom surface of TPS test piece is inappropriate, and was replaced with a TPS test piece in which the heat transfer coupled with convection and radiation occurred at the bottom surface of the test piece. An effective heat transfer coefficient $h_{\text {bottom }}$ is proposed and used to represent the combined heat transfer mode at the bottom surface of the TPS test piece. Here, the boundary condition at the bottom surface of TPS test piece is as follows:

$-\left.k_{i} \frac{\partial T(l, t)}{\partial z}\right|_{i=6}=h_{\text {bottom }}\left[T(l, t)-T_{s}\right]$

where $l$ is the total thickness of the TPS.

Combined with the actual heating conditions, the experimental heating load $T_{\mathrm{e}}$ was set as the black curve in Fig. 20. At this stage, the boundary condition at the top surface of the TPS test piece is as follows:

$T(0, t)=T_{\mathrm{e}}(t), t \in\left[0, t_{\mathrm{e}}\right]$

where $t_{\mathrm{e}}$ is the experimental heating time of $500 \mathrm{~s}$.

Five temperature sensors were installed at the center of the top surface and bottom surface of each test piece for temperature control and measurement. A schematic of the five temperature sensor locations is shown in Fig. 21. The mean value measured by the temperature sensors was taken as the test value.

The real heat loads from the first to the third experiment are 


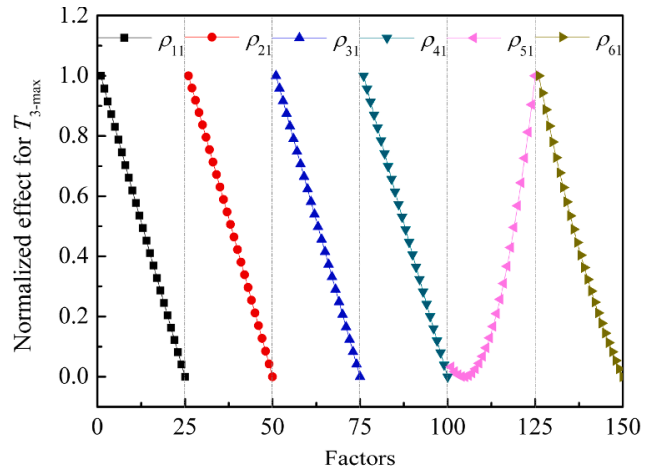

(a) $\rho$

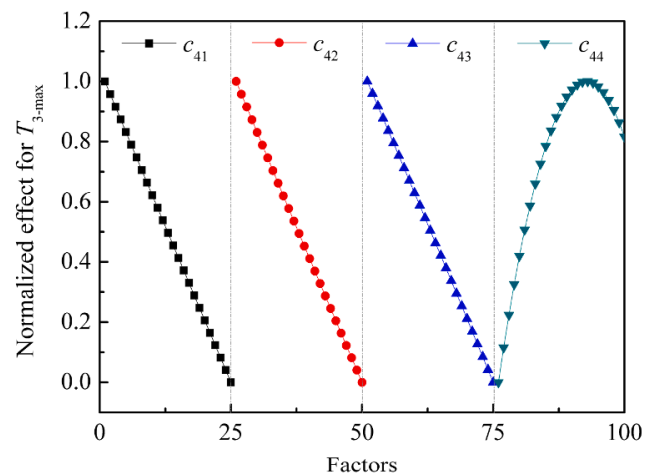

(c) $c_{4}$

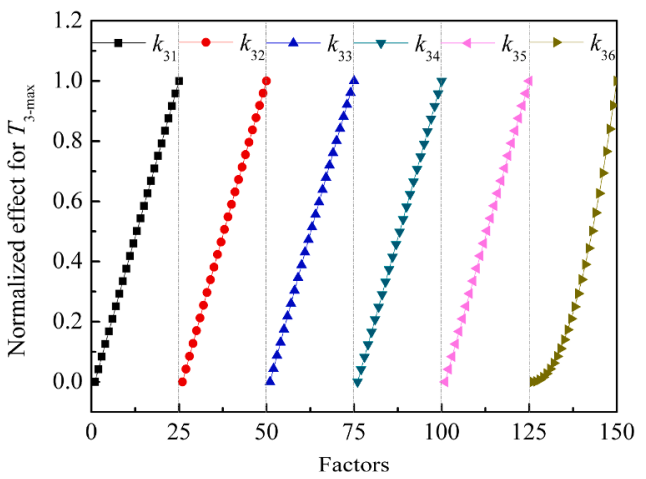

(g) $k_{3}$

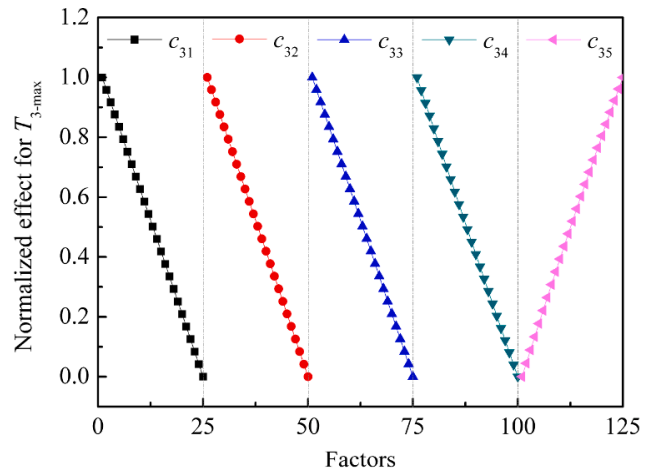

(b) $c_{3}$

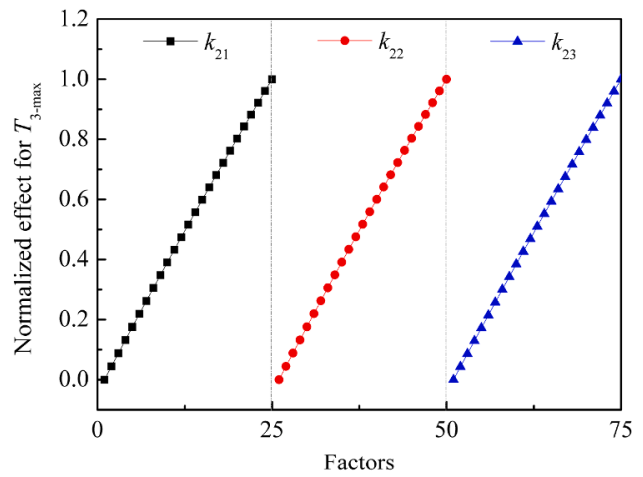

(d) $k_{2}$

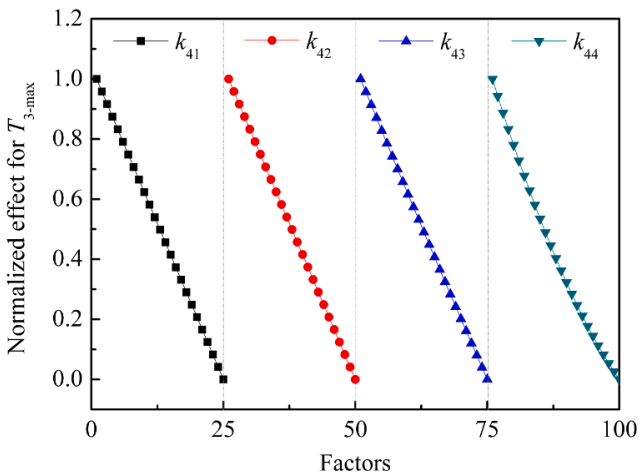

(h) $k_{4}$

Fig. 16. Effects of uncertainty parameter trends on $T_{3-\max }$.

illustrated by the red, green, and blue dots in Fig. 20. The first and second experiments take the initial test piece as the object, while the third experiment takes the final test piece as the object. Compared with the set value, actual heat loads have slight and acceptable deviations. It is worth noting that the vast deviation at the initial stage of heating resulted from the display range limitation of the equipment, and does not indicate the actual deviation. The real heating process of TPS the test piece is shown in Fig. 22.

After $500 \mathrm{~s}$, heating was stopped and the heat source was removed. At this stage, the boundary condition at the top surface of TPS test piece was changed to the following equation:

$$
\begin{aligned}
- & \left.k_{i} \frac{\partial T(0, t)}{\partial z}\right|_{i=1} \\
& =-h_{\text {top }}\left[T(0, t)-T_{s}\right]-\varepsilon(T) \sigma\left[T^{4}(0, t)-T_{s}^{4}\right], t \in\left[t_{\mathrm{e}}, t_{\mathrm{c}}\right]
\end{aligned}
$$

where $t_{\mathrm{c}}$ is the numerical calculation time of $6000 \mathrm{~s}$; and $h_{\text {top }}$, as shown in Fig. 23, is a natural convection heat transfer coefficient, which was calculated by the following empirical formulas $[35,36]$ :

$N u=\frac{h_{\text {top }} l}{k}=C\left(G_{r} P_{r}\right)_{m}^{n}$

where $N u$ is the Nusselt number; $l$ is the height of the top surface of the TPS test piece when placed vertically; $k$ is the thermal conductivity of air; $G_{r}$ is the Grashof number; $P_{r}$ is the Prandtl number; $C$ and $n$ are constants depending on the flow state of the natural convection, whose values are taken according to Ref. [36].

Except for the above boundary conditions, the governing conservation of energy equation under the experimental condition is the same as that under the re-entry condition.

\subsection{Uncertainty-based model updating and validation}

Owing to the unavoidable differences between the results of the numerical model and test data, model updating must be carried out to 


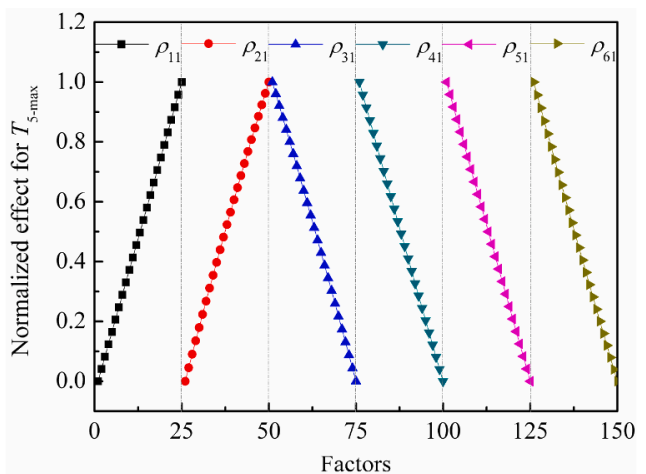

(a) $\rho$

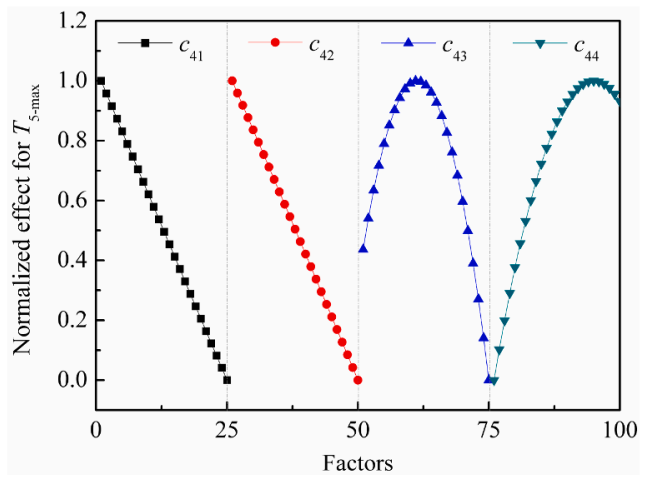

(c) $c_{4}$

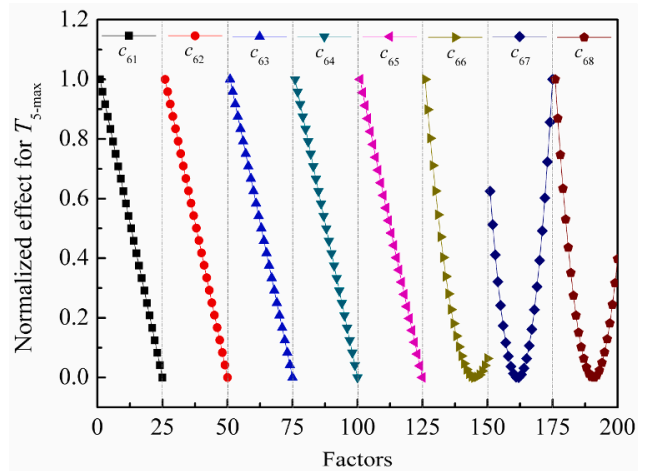

(e) $c_{6}$

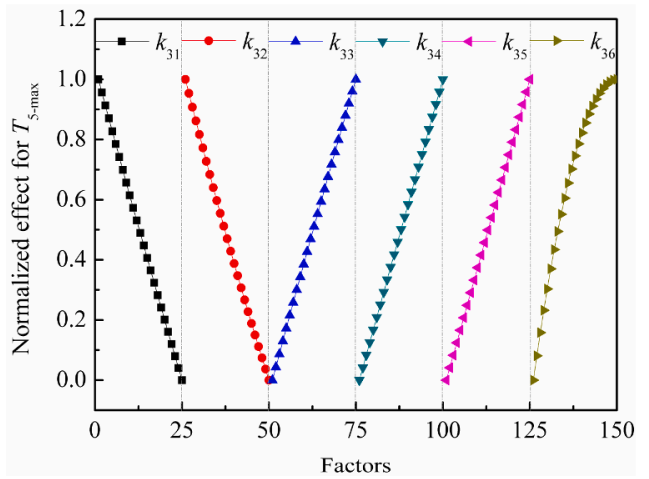

(g) $k_{3}$

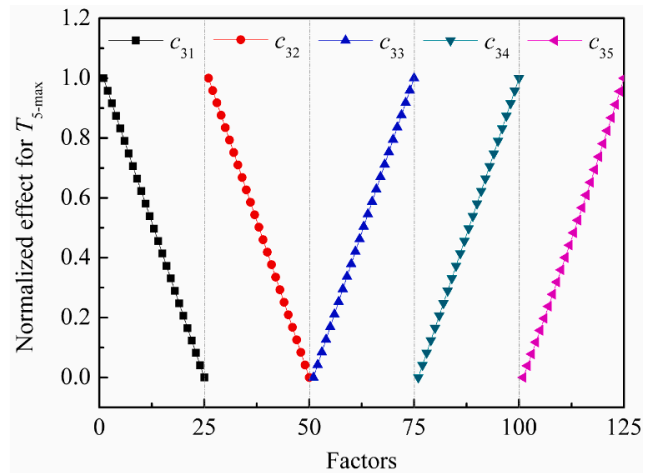

(b) $c_{3}$

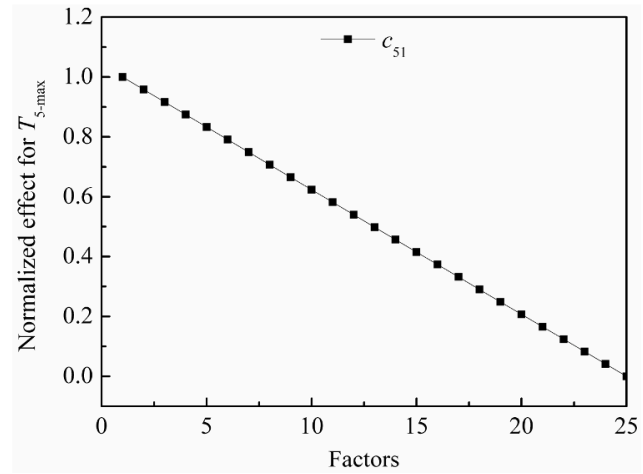

(d) $c_{5}$

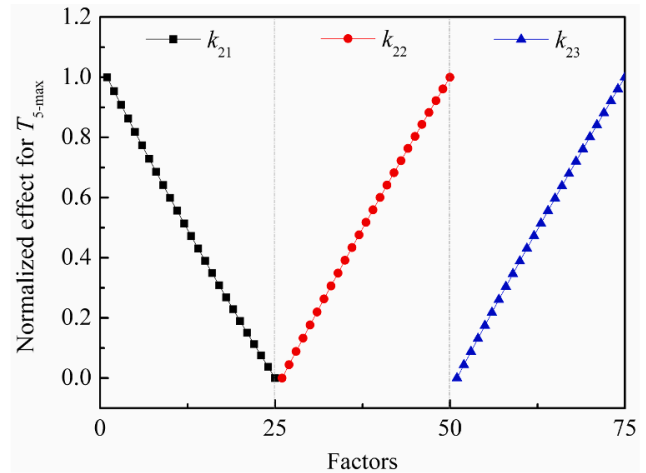

(f) $k_{1}$

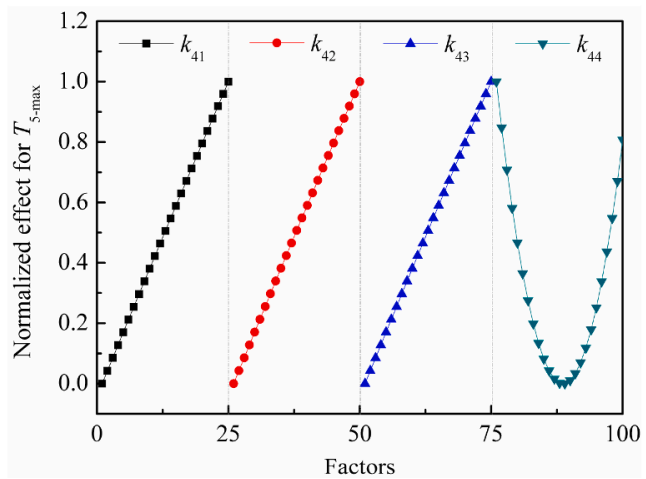

(h) $k_{4}$

Fig. 17. Effects of uncertainty parameter trends on $T_{5-\max }$.

bring the finite element model for the TPS unit closer to the actual test piece.

Model updating usually minimizes errors between the numerical model and the real tested structure by optimization. The single objective function method (SOF) was applied to the uncertainty-based finite element model updating. For the TPS, the skin's maximum temperature and its corresponding time are essential to the thermal insulation performance design. Hence, the objective function is to minimize the weighted sum of squares of the normalized residual of the interval median of the TPS bottom surface's maximum temperature and its 
Table 3

Bounds of the uncertainty responses.

\begin{tabular}{llll}
\hline$T_{3-\max }(\mathrm{K})$ & $A_{1}=\left[\begin{array}{lll}553.50, & 645.47\end{array}\right]$ & $B_{1}=\left[\begin{array}{lll}555.72, & 643.11\end{array}\right]$ & $C_{1}=\left[\begin{array}{ll}572.91, & 627.78\end{array}\right]$ \\
\hline$T_{5-\max }(\mathrm{K})$ & $A_{2}=\left[\begin{array}{lll}155.60, & 200.18\end{array}\right]$ & $B_{2}=\left[\begin{array}{lll}157.14, & 197.97\end{array}\right]$ & $C_{2}=\left[\begin{array}{lll}164.23, & 190.17\end{array}\right]$ \\
\hline
\end{tabular}

corresponding time. The effective heat transfer coefficient $h_{\text {bottom }}$ was selected as the only design variable to be updated. Finally, an uncertainty-based model updating method for the heat transfer analysis and design of TPS was proposed, and shown in the way of an optimization model as follows:

$$
\begin{gathered}
\text { find } h_{\text {bottom }} \\
\min \omega_{1}\left(\frac{T_{6-\max }^{\mathrm{FEM}-\mathrm{MED}}\left(h_{\mathrm{bottom}}\right)-T_{6-\max }^{\mathrm{EXP}}}{T_{6-\max }^{\mathrm{EXP}}}\right)^{2}+\omega_{2}\left(\frac{t_{T_{6}-\max }^{\mathrm{EEM}-\mathrm{MED}}\left(h_{\mathrm{bottom}}\right)-t_{T_{6}-\operatorname{mXx}}^{\mathrm{EXP}}}{t_{T_{6-\max }}^{\mathrm{EP}}}\right)^{2} \\
\text { s. t. } \omega_{1}+\omega_{2}=1 \\
\omega_{1}, \omega_{2} \geqslant 0
\end{gathered}
$$

where $T_{6-\max }^{\mathrm{FEM}-\mathrm{MED}}$ and $t_{T_{6-\max }}^{\mathrm{FEM}}$ are the interval median of the maximum temperature and corresponding time of the TPS bottom surface during finite element analysis, respectively. $T_{6-\max }^{\mathrm{EXP}}$ and $t_{T_{6-\max }}^{\mathrm{EXP}}$ are the maximum temperature and the corresponding time of the TPS bottom surface for test data, respectively. $\omega_{1}$ and $\omega_{2}$ are weighting factors and were set to be equal.

The updated effective heat transfer coefficient was solved as $h_{\text {bottom }}=1.75 \mathrm{~W} /\left(\mathrm{m}^{2} \cdot \mathrm{K}\right)$. The finite element results before and after model updating and their comparison with test data are shown in Fig. 24. The red curve describes the nominal temperature history of the TPS bottom surface when $h_{\text {bottom }}=0$ during finite element analysis, which corresponds with the assumption that no heat transfer occurs at the bottom surface of TPS. The blue curve represents the nominal temperature history of the TPS bottom surface when $h_{\text {bottom }}=1.75 \mathrm{~W} /\left(\mathrm{m}^{2} \cdot \mathrm{K}\right)$ during finite element analysis. The black curve represents the experimental temperature history of the TPS bottom surface. It can be seen that the nominal values of maximum temperature and the corresponding time of TPS bottom surface after the uncertainty-based model updating are much closer to the test data, which proves the validity of the uncertainty-based model updating method. Meanwhile, the heat transfer model of the TPS test piece was validated. The temperature history of the TPS top surface after model updating is shown in Fig. 25.

\subsection{Experimental verification}

Using the updated finite element model of the test piece, uncertainty heat transfer analysis based on ILHD for $T_{6-\max }$ was carried out. The distribution domains of the uncertainty analysis and test data

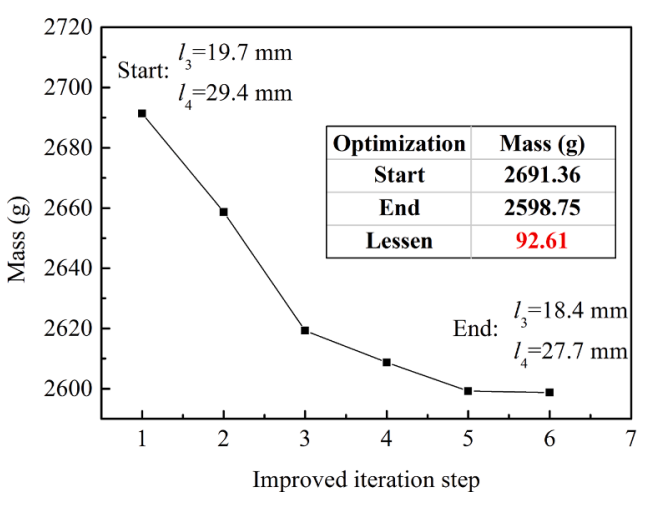

(a) Mass

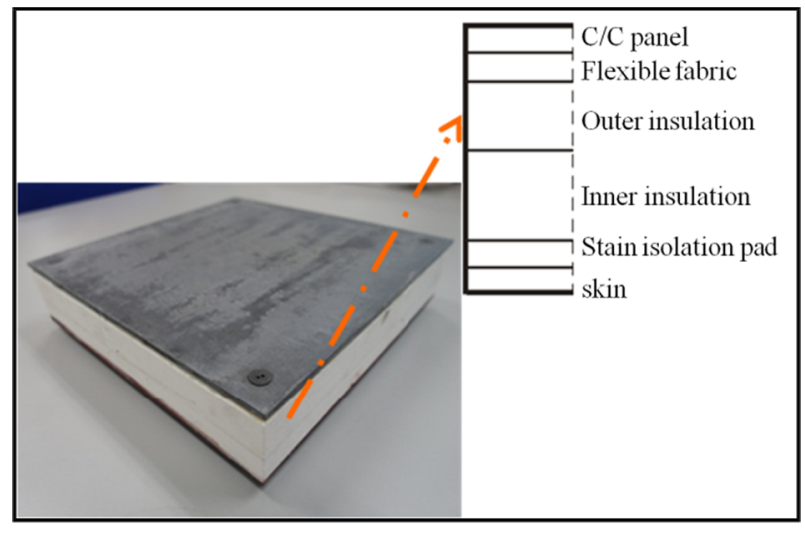

Fig. 19. Test piece.

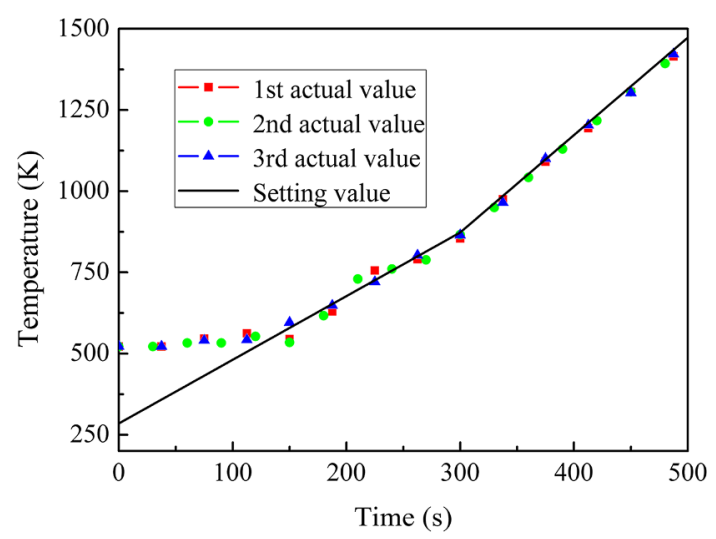

Fig. 20. Set heat load and three actual heat loads.

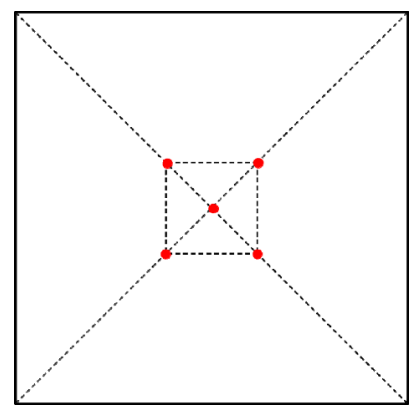

Fig. 21. Schematic of five temperature sensor locations.

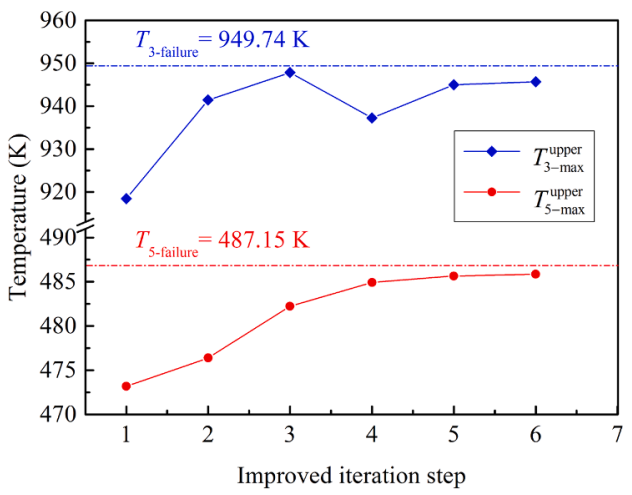

(b) Constrained responses

Fig. 18. Iteration history. 


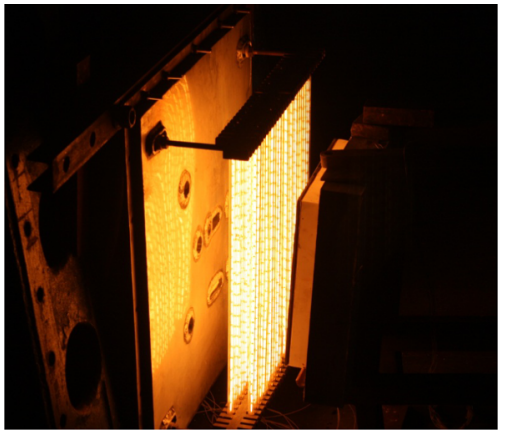

Fig. 22. Heating process.

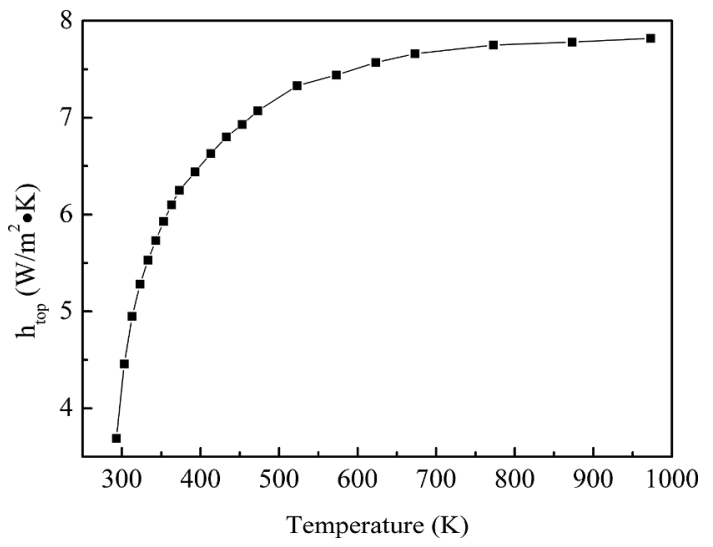

Fig. 23. Convection heat transfer coefficient of the top surface of test piece.

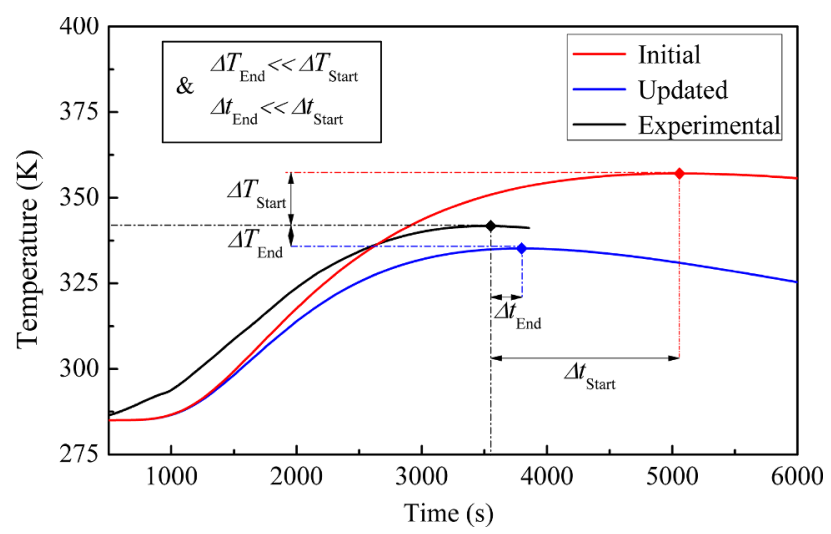

Fig. 24. Temperature histories of TPS bottom surface.

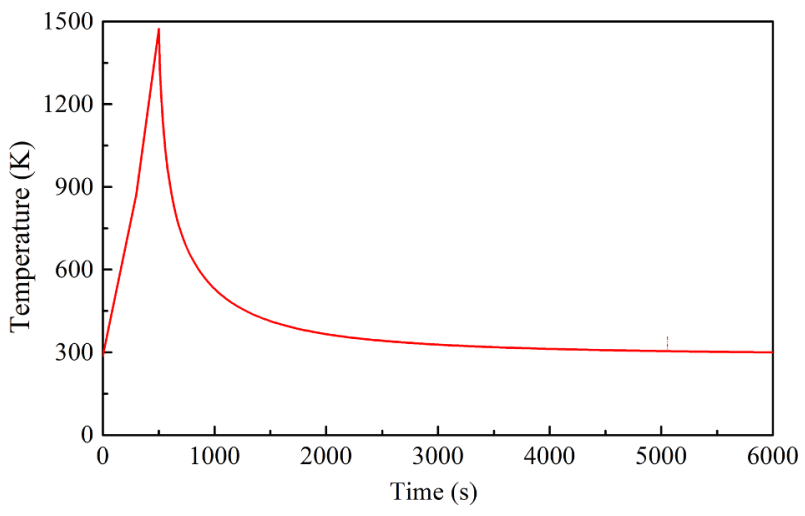

Fig. 25. Temperature history of TPS top surface after model updating.
Table 4

Bounds and test value of the $T_{6-\max }$.

\begin{tabular}{llll}
\hline Test Piece & Lower Bound & Upper Bound & Test Value \\
\hline Initial Piece & $327.62 \mathrm{~K}$ & $351.60 \mathrm{~K}$ & $341.40 \mathrm{~K}$ \\
Final Piece & $334.54 \mathrm{~K}$ & $354.65 \mathrm{~K}$ & $347.15 \mathrm{~K}$ \\
\hline
\end{tabular}

\section{Deterministic design}

\section{Uncertainty based design}

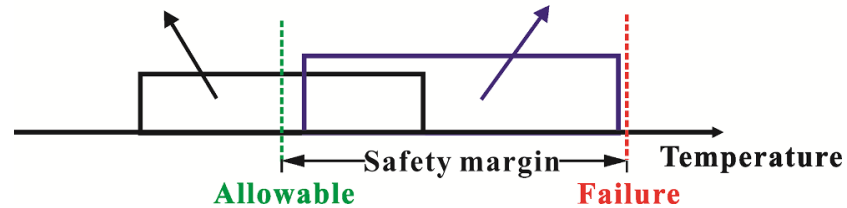

Fig. 26. Reliability schematic of deterministic design and uncertainty-based design.

for $T_{6-\max }$ are presented in Table 4. For the initial test piece, the test value is in the distribution domain of uncertainty analysis, which proves the correctness of the uncertainty analysis based on ILHD. For the final test piece, the test value is still within the distribution domain of uncertainty analysis, which proves the correctness of uncertainty optimization design.

The correctness of the uncertainty analysis and optimization design for the TPS has been verified. Moreover, the response distribution domains of uncertainty analysis based on ILHD all contain those obtained by other methods, as shown in Section 3.2, which proves the effectiveness of the uncertainty analysis based on ILHD. The TPS mass by nonprobabilistic optimization is $3.44 \%$ lighter compared with that by deterministic optimization. The former also maintains the same reliability as the latter, as shown in Fig. 26, which proves the effectiveness of uncertainty optimization design. Finally, a lighter TPS design was obtained, and both the correctness and validity of the applied methods were verified.

\section{Conclusions}

This paper presented a complete uncertainty-based TPS design process that includes deterministic heat transfer analysis, sensitivity and uncertainty analyses, nonprobabilistic optimization, and experimental verification.

Firstly, an ILHD was proposed. It is an advanced method not only in the field of sensitivity and uncertainty analyses but also in the area of computer experiment design, with the ability to consider the correlations between uncertainty parameters, have good space-filling, and fit the nonlinear response well. Sensitivity and uncertainty analyses using ILHD has both high accuracy and low computational cost. The TPS weight by uncertainty-based design is $3.44 \%$ lighter compared with that by deterministic design, which indicates that the uncertainty-based design offers new ideas for structural weight loss. An uncertainty-based finite element model updating method to modify the heat transfer numerical model of the TPS was also proposed and applied, which provides some guidance for future experimental verification.

In summary, the TPS designed by the proposed uncertainty-based methods has the advantages of adequate thermal insulation and light weight compared with traditional design, and shows excellent potential for applications in the development of high-performance hypersonic vehicles.

\section{Replication of results}

The raw data required to reproduce these findings are available in the figures and tables of the paper. 


\section{Declaration of Competing Interest}

The authors declare that they have no known competing financial interests or personal relationships that could have appeared to influence the work reported in this paper.

\section{Acknowledgments}

The work of this paper is supported by the National Nature Science Foundation of China (No. 11872089, 11902322), the Defense Industrial Technology Development Program (Nos. JCKY2017601B001, JCKY2017208B001, JCKY2019203A003), the National Science and Technology Major Project (2017-IV-0010-0047).

\section{References}

[1] T. Ji, R. Zhang, B. Sunden, et al., Investigation on thermal performance of high temperature multilayer insulations for hypersonic vehicles under aerodynamic heating condition, Appl. Therm. Eng. 70 (1) (2014) 957-965.

[2] K. Daryabeigi, Thermal analysis and design of multi-layer insulation for re-entry aerodynamic heating, 35th AIAA Thermophysics Conference, 2001, p. 2834.

[3] Y.K. Chen, T. Squire, B. Laub, et al., Monte Carlo analysis for spacecraft thermal protection system design, 9th AIAA/ASME Joint Thermophysics and Heat Transfer Conference, (2006).

[4] S. Chiu, W. Pitts, Reusable surface insulations for reentry spacecraft, 29th Aerospace Sciences Meeting, 1991, p. 695.

[5] D. Glass, Ceramic matrix composite (CMC) thermal protection systems (TPS) and hot structures for hypersonic vehicles, 15th AIAA International Space Planes and Hypersonic Systems and Technologies Conference, (2008).

[6] J.J. Bertin, R.M. Cummings, Fifty years of hypersonics: where we've been, where we're going, Prog. Aerosp. Sci. 39 (6/7) (2003) 511-536.

[7] D. Myers, C. Martin, M. Blosser, Parametric weight comparison of current and proposed thermal protection system (TPS) concepts, 33rd Thermophysics Conference, 1999, p. 3459.

[8] D. Li, Heat transfer characteristics of high temperature multilayer thermal insulations, Aerosp. Mater. Technol. 41 (1) (2011) 20-23.

[9] M.J. Wright, D. Bose, Y.K. Chen, Probabilistic modeling of aerothermal and thermal protection material response uncertainties, AIAA J. 45 (2) (2007) 399-410.

[10] Chenyu Cao, Ruixing Wang, Xiaodong Xing, Wenfeng Liu, Hongwei Song, Chenguang Huang, Performance improvement of integrated thermal protection system using shaped-stabilized composite phase change material, Appl. Therm. Eng. 164 (2020), https://doi.org/10.1016/j.applthermaleng.2019.114529 ISSN 13594311.

[11] F. Gori, S. Corasaniti, W.M. Worek, et al., Theoretical prediction of thermal conductivity for thermal protection systems, Appl. Therm. Eng. 49 (2012) 124-130.

[12] Y. Ma, B. Xu, M. Chen, et al., Optimization design of built-up thermal protection system based on validation of corrugated core homogenization, Appl. Therm. Eng. 115 (Complete) (2017) 491-500.

[13] W. Li, H. Huang, B. Ai, et al., On the novel designs of charring composites for thermal protection application in re-entry vehicles, Appl. Therm. Eng. 93 (2016) 849-855.

[14] X.J. Wang, Q. Ren, W.P. Chen, Structural design optimization based on the moving baseline strategy, Acta Mech. Solida Sin. 33 (2020) 307-326.
[15] P.A. Gnoffo, K.J. Weilmuenster, H.H. Hamilton, et al., Computational aerothermodynamic design issues for hypersonic vehicles, J. Spacecraft Rockets 36 (1) (1999) 21-43.

[16] A. Gomez-San-Juan, I. Perez-Grande, A. Sanz-Andres, Uncertainty calculation for spacecraft thermal models using a generalized SEA method, Acta Astron. 151 (Oct.) (2018) 691-702.

[17] ECSS Secretariat, Space Engineering - Thermal Analysis Handbook, ECSS-EHB-3103A, Noordwijk, The Netherlands, 2016.

[18] J. Dec, R. Mitcheltree, Probabilistic design of a Mars Sample Return Earth entry vehicle thermal protection system, 40th AIAA Aerospace Sciences Meeting \& Exhibit, 2002, p. 910.

[19] B.A. Zárate, J.M. Caicedo, Finite element model updating: Multiple alternatives, Eng. Struct. 30 (12) (2008) 3724-3730.

[20] K. Sun, Y. Zhao, H. Hu, Identification of temperature-dependent thermal-structural properties via finite element model updating and selection, Mech. Syst. Sig. Process. 52-53 (1) (2015) 147-161.

[21] Hua-Peng Chen, Yi-Qing Ni, Finite element model updating, Structural Health Monitoring of Large Civil Eng. Struct. (2018).

[22] I. Torralbo, I. Perez-Grande, A. Sanz-Andres, et al,, Correlation of spacecraft thermal mathematical models to reference data, Acta Astron. (2017) S0094576517313334.

[23] I. Torralbo, A. Sanz-Andres, J. Piqueras, et al., Correlation of Thermal Mathematical Models to test data using Jacobian matrix formulation, 48th International Conference on Environmental Systems, (2018).

[24] Gongnan Xie, Qi Wang, Bengt Sunden, et al., Thermomechanical optimization of lightweight thermal protection system under aerodynamic heating, Appl. Therm. Eng. 59 (1-2) (2013) 425-434.

[25] C. Yang, X. Hou, L. Wang, Thermal design, analysis and comparison on three concepts of space solar power satellite, Acta Astronaut. (2017).

[26] W.L. Ko, R.D. Quinn, L. Gong, et al., Re-entry heat transfer analysis of the space shuttle orbiter, NASA CP-2216, 1982, pp. 295-325.

[27] B. Ravishankar, Deterministic and reliability based optimization of integrated thermal protection system composite panel using adaptive sampling techniques, Health Policy 106 (1) (2012) 1-2.

[28] C. Wang, Z. Qiu, M. Xu, Collocation methods for fuzzy uncertainty propagation in heat conduction problem, Int. J. Heat Mass Transf. 107 (2017) 631-639.

[29] L. Wang, X.J. Wang, Y.L. Li, J.X. Hu, A non-probabilistic time-variant reliable control method for structural vibration suppression problems with interval uncertainties, Mech. Syst. Sig. Process. 115 (2019) 301-322.

[30] C. Yang*, K. Liang, X.P. Zhang, Strategy for sensor number determination and placement optimization with incomplete information based on interval possibility model and clustering avoidance distribution index, Comput. Methods Appl. Mech. Eng. 366 (2020).

[31] X.J. Wang, Q.H. Shi, W.C. Fan, R.X. Wang, L. Wang, Comparison of the reliabilitybased and safety factor methods for structural design, Appl. Math. Model. 72 (2019) 68-84.

[32] E. Borgonovo, A new uncertainty importance measure, Reliab. Eng. Syst. Saf. 92 (6) (2017) 771-784.

[33] Q.H. Shi, X.J. Wang, W.P. Chen, K.J. Hu, Optimal sensor placement method considering the importance of structural performance degradation for the allowable loadings for damage identification, Appl. Math. Model. 86 (2020) 384-403, https:// doi.org/10.1016/j.apm.2020.05.021.

[34] A. Saltelli, Sensitivity analysis for importance assessment, Risk Anal. Off. Public Soc. Risk Anal. 22 (3) (2002) 579-590.

[35] F.P. Incropera, Fundamentals of Heat and Mass Transfer, Katson, 1985.

[36] S.M. Yang, W.Q. Tao, Heat Transfer, 4rd, Higher Education Press, Beijing, 2006 (in Chinese). 